\title{
The Effect of Electrospun Fiber Diameter on Astrocyte-Mediated Neurite Guidance and Protection
}

\author{
Christopher D. L. Johnson ${ }^{\dagger, \ddagger}$, Jonathan M. Zuidema ${ }^{\S}$, Kathryn R. Kearns ${ }^{\dagger, \ddagger}$, Alianna B. \\ Maguire $^{\ddagger}$, Gregory P. Desmond ${ }^{\ddagger}$, Deanna M. Thompson ${ }^{\dagger} \neq$, and Ryan J. Gilbert ${ }^{\star}, \dagger \neq$ \\ ${ }^{\dagger}$ Center for Biotechnology and Interdisciplinary Studies, Rensselaer Polytechnic Institute, 110 \\ Eighth Street, Troy, New York 12180-3590, United States \\ ‡Department of Biomedical Engineering, Rensselaer Polytechnic Institute, 110 Eighth Street, Troy, \\ New York 12180-3590, United States \\ $\S$ Department of Chemistry and Biochemistry, University of California San Diego, 9500 Gilman Dr., \\ La Jolla, California 92093, United States
}

\begin{abstract}
The topography of electrospun fiber scaffolds modifies astrocytes toward in vivo-like morphologies and behaviors. However, little is known about how electrospun fiber diameter influences astrocyte behavior. In this work, aligned fibers with two distinct nanoscale fiber diameters (808 and $386 \mathrm{~nm}$ ) were prepared, and the astrocyte response was measured over time. Astrocytes on the large diameter fibers showed significantly increased elongation as early as $2 \mathrm{~h}$ after seeding and remained significantly more elongated for up to 4 days compared to those on small diameter fibers. Astrocytes extending along larger diameter fibers were better equipped to support long neurite outgrowth from dorsal root ganglia neurons, and neurite outgrowth along these astrocytes was less branched than outgrowth along astrocytes cultured on small diameter fibers. The differences in astrocyte shape observed on the small or large diameter fibers did not translate into differences in GLT-1, GFAP, or GLAST protein expression. Thus, different fiber diameters were unable to influence astrocyte protein expression uniquely. Nevertheless, astrocytes cultured in either small or large fibers significantly increased their expression of GLT-1 compared to astrocytes cultured on nonfiber (film) controls. Fibrous-induced increases in astrocyte GLT-1
\end{abstract}

\footnotetext{
This article is made available for a limited time sponsored by ACS under the ACS Free to Read License, which permits copying and redistribution of the article for non-commercial scholarly purposes.

"Corresponding Author: gilber2@ rpi.edu.

Author Contributions

The manuscript was written through contributions of all authors. All authors have given approval to the final version of the manuscript. The authors declare no competing financial interest.

Supporting Information

The Supporting Information is available free of charge on the ACS Publications website at DOI: 10.1021/acsabm.8b00432.

(Figure S1) Analysis of fiber diameter as the PLLA concentration is varied from 6 to 12\%, and analysis of fiber diameter distribution between the small and large fibers; (Figure S2) analysis of electrospun fiber alignment as the PLLA concentration is varied from 6 to 12\%; (Figure S3) contact angle hysteresis on small fiber, large fiber, and film surfaces, (Figure S4) preliminary experiment to determine early time points of astrocyte adhesion and elongation; (Figure S5) immunocytochemistry analysis of Actin, GFAP, GLT-1, and DAPI in astrocytes cultured on small fiber, large fiber, and film surfaces for 2, 6, 24, or 96 h; (Figure S6) additional neurite tracing data from Astrocyte/Neuron coculture: longest neurite, total length, branch points, and length per primary neurite; (Figure S7) Western blot analysis of astrocyte GFAP expression on small fiber, large fiber, and film surfaces after 1 or 4 days; (Figure S8) neurononly and astrocyte-only controls for the glutamate excitotoxicity assay (PDF)
} 
expression protected astrocyte/neuron cocultures from toxicity generated by high extracellular glutamate. Alternatively, astrocytes/neurons cultured on films were less able to protect these cells from culture conditions consisting of high glutamate levels. Biomaterials, such as the fibrous materials presented here, may help stimulate astrocytes to increase GLT-1 expression and uptake more glutamate, since astrocytes are less likely to uptake glutamate in neurodegenerative pathologies or following central nervous system injury.

\section{Graphical Abstract}

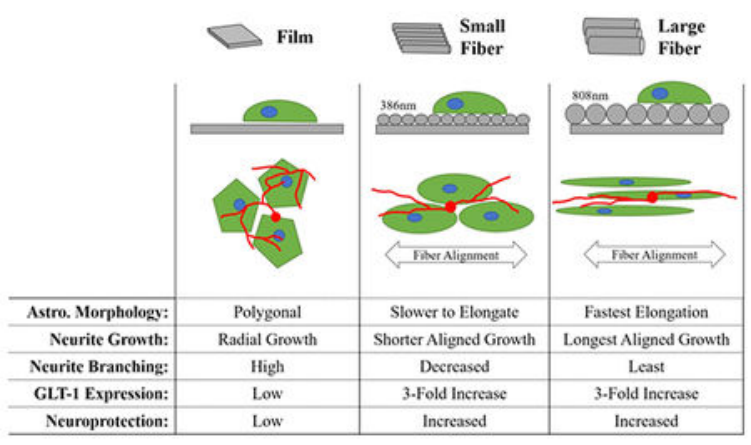

\section{Keywords}

electrospun fiber diameter; astrocyte; neuron; glutamate uptake; topographical guidance; neuroprotection

\section{INTRODUCTION}

Astrocytes are important therapeutic targets in the brain and spinal cord because of their ability to maintain homeostasis in the healthy central nervous system (CNS) and mitigate the severity of injury. ${ }^{1-4}$ In the healthy CNS, astrocytes also transport nutrients to neurons and glia, maintain the blood-brain barrier, and regulate neurotransmitter clearance. ${ }^{2,3,5}$ After injury, astrocytes work to re-establish homeostasis by restoring the blood-brain barrier, removing excitotoxic glutamate, neutralizing reactive oxygen species, releasing neuroprotective adenosine, protecting from ammonia toxicity, and reducing edema (reviewed by Sofroniew and Vinters ${ }^{5}$ ). Approaches that modify astrocyte behavior may provide insight into treatment strategies for CNS injury and disease.

Astrocytes respond to injury by undergoing rapid and distinct morphological and biochemical changes, known collectively as astrocyte reactivity (reviewed in refs 1-5). The astrocyte response to injury exists along a spectrum, ${ }^{1}$ but astrocyte reactivity and the scarring response were thought to be irreversible. ${ }^{6,7}$ Recently, however, Hara and colleagues observed that astrocyte reactivity was both reversible and dependent on the surrounding environment. ${ }^{8}$ Therefore, biomaterial approaches that modify the injury environment have the potential to reduce astrocyte reactivity after CNS injury, a theory that is supported by the literature: Mattotti and colleagues observed that specific dimensions of aligned grooves on poly(methyl methacrylate) PMMA surfaces triggered mature astrocytes to revert back toward a radial-glia-like state. ${ }^{9}$ Similarly, the geometry of electrospun fiber scaffolds shifted 
astrocytes toward behaviors that supported neuron viability and process extension. ${ }^{10-16}$ While studies have identified certain physical properties of the scaffold that alter astrocyte morphology (such as fiber alignment ${ }^{13,16}$ and fiber surface nanotopography ${ }^{17}$ ), the influence of fiber diameter on astrocyte behavior is still not fully understood.

Neurons and glia respond differently when electrospun fiber diameters are larger than 400$500 \mathrm{~nm}$. Fiber diameters between 500 and $5000 \mathrm{~nm}$ promote the longest directed extension of neurites from a neural explant culture model (dorsal root ganglion (DRG)). ${ }^{18-20}$ Fiber diameters larger than $400 \mathrm{~nm}$ stimulate increased myelination by oligodendrocytes. ${ }^{21}$ The $^{2}$ fiber diameter also dictates the length and thickness of the myelin sheath. ${ }^{22}$ The apparent threshold around 400-500 $\mathrm{nm}$ may be related to the scale of natural fibrous features found in the native CNS. Myelinated axons can reach diameters in excess of $20 \mu \mathrm{m},{ }^{23,24}$ and the average mature myelinated axon is more than twice the diameter of the ECM (1000-5000 $\mathrm{nm}) .{ }^{25,26}$ Fibrous elements in the extracellular matrix (ECM) range in diameter from 50 to $500 \mathrm{~nm},{ }^{27-29}$ and the caliber of immature axons range from and $80-400 \mathrm{~nm} .^{23}$ These references suggest that fiber diameters larger than 400-500 $\mathrm{nm}$ mimic maturing axons that are distinct from the ECM matrix. However, little is known about how fiber diameters in this range influence astrocyte behavior.

One previous study found that increasing the fiber diameter in unaligned fiber scaffolds restricted astrocyte spreading. ${ }^{30}$ However, the study did not use aligned fiber scaffolds or examine changes in astrocyte phenotype-like the examination of glial fibrillary acidic protein (GFAP) production or glutamate transporter expression. No study has analyzed the ability of fiber diameter to alter astrocyte behavior when fibers are highly aligned. This is an important distinction because fiber alignment promotes longer, more-directed astrocyte migration and extension of astrocyte processes 16,31 and improves the bridging response of neurons and astrocytes in vivo. ${ }^{31}$ We hypothesized that for aligned electrospun fibers, increases in fiber diameter would result in increased astrocyte elongation. We then studied if fiber-induced changes in astrocyte shape influenced the astrocyte's ability to direct extending neurites from dorsal root ganglia neurons and if these changes in astrocyte shape influenced the ability of astrocytes to produce several key biomarkers associated with reactivity and neuroprotection.

Aligned, electrospun fiber scaffolds trigger astrocytes to upregulate their expression of transporters of glutamate and facilitate the uptake of glutamate. Of the excitatory neurotransmitters produced by CNS glia and neurons, glutamate, in high extracellular concentrations induces neuronal excitotoxicity. Astrocytes remove glutamate from the extracellular environment using two glutamate transporters: excitatory amino acid transporters 1 and 2 (EAAT-1 and EAAT-2), ${ }^{32}$ the homologues for which in rats are GLAST and GLT-1, respectively. ${ }^{33}$ When compared to astrocytes seeded on flat polymer films, astrocytes seeded on electrospun fibers with diameters of $2400 \mathrm{~nm}$ significantly increased expression of GLT-1 glutamate transporters, but not GLAST. ${ }^{16}$ Astrocytes cultured on fibers took up more glutamate than astrocytes cultured on films. ${ }^{16}$ Since glutamate uptake has been linked to astrocyte morphology through Rho Kinase (ROCK)-mediated actin dynamics, ${ }^{34,35}$ we hypothesized that changes in astrocyte morphology on smaller diameter fibers would result in decreased GLT-1 expression. 
We believed that increasing the diameter of electrospun fibers above $500 \mathrm{~nm}$ would heighten astrocyte extension, increase astrocyte-induced neurite elongation, and increase astrocyte expression of glutamate transporters, in particular GLT-1. These hypotheses were tested by culturing astrocytes on flat films and aligned electrospun fibers with either large ( $808 \mathrm{~nm})$ or small $(386 \mathrm{~nm})$ diameters. Astrocyte morphology was measured on each scaffold type at distinct time points after seeding to examine how quickly astrocytes responded to small diameter or large diameter topography. We then investigated if the astrocyte morphological changes observed on the surfaces influenced the extent and direction of neurite outgrowth seeded on top of the established astrocyte cultures. Next, GLT-1, GLAST, and glial fibrillary acidic protein (GFAP) expression were measured on the different surfaces using Western blot. Finally, cellular viability of cocultures of astrocytes and neurons were assessed when excitotoxic levels of extracellular glutamate were added to the cultures to examine whether increased GLT-1 or GLAST production enabled neuroprotection.

\section{MATERIALS AND METHODS}

\subsection{Scaffold Preparation}

2.1.1. Film Casting-Fibers were electrospun onto a (poly-L-lactic acid) PLLA film to reduce cell interactions with materials other than PLLA and to affix the fibers to the glass coverslip surface using methods reported previously. ${ }^{18}$ PLLA (4 wt \%/wt; Cargill Dow LLC, Minnetonka, MN) was placed in a solution consisting of chloroform and dichloromethane (1 to 1 [wt/wt] solution; Sigma-Aldrich, St. Louis MO) and solution cast onto glass coverslips with dimensions of $15 \mathrm{~mm}$ by $15 \mathrm{~mm}$ (Knittel Glass, Brausenweig, Germany). The solution was placed onto the coverslips via a $5 \mathrm{~mL}$ syringe, and the solution was placed onto the surface drop by drop until the solution covered the coverslip. The solvent was allowed to evaporate at room temperature (RT) overnight, leaving a flat PLLA film on the coverslip.

2.1.2. Fabrication of Small and Large PLLA Nanofibers-Aligned electrospun fibers were prepared using previously described methods ${ }^{36}$ and using the parameters specified in Table 3. Fibers were collected on PLLA-coated glass coverslips, rotating on a 22 $\mathrm{cm}$ diameter mandrel. PLLA solution was delivered with a 22-gauge needle purchased from BD Biosciences (Franklin Lakes, NJ) at $2.03 \mathrm{~mL} \mathrm{~h}^{-1}$ across a $5 \mathrm{~mm}$ gap using a power supply from Gamma High Voltage Research (Orlando, FL) set to $10 \mathrm{kV}$.

Control of fiber diameter occurred by altering the PLLA content in solution (see Figure S1). Increasing the weight percent of polymer molecules increases the number of chain entanglements and increases the fiber diameter. ${ }^{37}$ Electrospun fibers with smaller diameters were prepared using a 7\% solution (wt of PLLA per wt of 1,1,1,3,3,3-hexafluoro-2-propanol (HFP; Sigma)), and electrospun fibers with larger diameters were fabricated using a $12 \%$ solution (wt of PLLA per wt of HFP). The fibers were fluorescently labeled by adding $2 \mu \mathrm{L}$ of rhodamine B (1 mg/mL in dimethyl sulfoxide (DMSO), Sigma) to the electrospinning solution.

2.1.3. Imaging of PLLA Nanofibers-Scanning electron microscopy (SEM) was used to quantify differences in fiber physical properties like fiber alignment, density, and diameter for small and large diameter fibers. Samples were coated with $5 \mathrm{~nm}$ of platinum using a 
Technics Hummer V sputter coater, and a Versa 3D SEM (FEI, Hillsboro, OR) was used to image the fiber scaffolds. Images were taken using an in-lens secondary electron detector with a $10 \mathrm{~mm}$ working distance, $20 \mu \mathrm{m}$ aperture, and $2 \mathrm{kV}$ acceleration voltage. Five images were taken at four corners and in the center of the coverslip for fiber characterization analysis.

\subsection{Fiber Characterization}

2.2.1. Fiber Diameter-Fiber diameter was measured with FIJI software (NIH, Bethesda, MD), from at least 100 fibers each from three independently fabricated samples. Fiber diameter was reported as mean \pm standard error.

2.2.2. Fiber Alignment-The line tool in FIJI software was used to measure the angle of the long axis of each fiber. Using the SEM images, 100 fibers' angles were measured from each scaffold fabricated independently $(N=3)$. The mean fiber alignment was calculated, and this value was used to determine the distribution of fiber alignment. Histograms were prepared with 0 indicating the mean alignment. Deviation angles were distributed into bins of $10^{\circ}$. Each graph (displayed as histograms) shows the entire population of fibers ( $>300$ measurements from $N=3$ scaffolds).

2.2.3. Fiber Density-The density was determined by counting the amount of fibers intersecting a $15 \mu \mathrm{m}$ bar oriented perpendicular to aligned fibers using FIJI software. At least 100 fibers were measured from scaffolds fabricated independently $(N=3)$. Graphs represent the average \pm the standard error of the mean.

2.2.4. Sca $\mathrm{fff}_{\mathrm{ff}} \mathrm{Old}$ Hydrophilicity-Surface hydrophilicity was measured to determine if differences in surface wettability influenced the results. Contact angle hysteresis analysis was conducted with a RameHart 500 goniometer (Succasunna, NJ) using a $1 \mu \mathrm{L}$ sessile water droplet. Hysteresis provides a more accurate representation of rough surfaces. The advancing and receding angles were analyzed using a LBADSA plugin in FIJI software (Lausanne, Switzerland). We determined the contact angle by taking the receding contact angle and subtracting it from the advancing contact angle. The graph represents the average values calculated from samples fabricated independently $(N=3)$.

\subsection{Primary Cell Culture}

\subsubsection{Isolation and Culture of Primary Cortical Rat Astrocytes-For these} experiments, we isolated primary astrocytes to study the effects of topographical fiber diameter on central nervous system glial cells. Since these fibers are constructed to interface with the spinal cord, we wanted to discern how astrocytes respond to fibers of differing diameter. We selected cortical astrocytes for these studies since in our previous publication, we only observed very subtle differences between spinal cord and cortical astrocytes. ${ }^{17}$ Primary cells isolated from Sprague-Dawley $\mathrm{p} 2$ neonatal rat cortices were isolated (Taconic Farms, Hudson, NY) using a protocol previously described. ${ }^{16}$ Cellular isolations from rats followed NIH guidelines, and isolations were authorized by the Institutional Animal Care and Use Committee (IACUC) at RPI. Care was taken to completely isolate the cortices from other parts of the brain during surgery. Cell removal from cortices transpired with three 
incubations (10 min each) in a 1:1 vol/vol solution of warmed $\left(37^{\circ} \mathrm{C}\right)$ TrypLE (Invitrogen)/ OptiMEM. The next incubation occurred with DNase I ( $1 \mathrm{mg} / \mathrm{mL}$; Sigma), and cell collection occurred after the DNase I treatment. A total of 200000 cells were delivered to each $1.0 \mu \mathrm{g} / \mathrm{mL}$ poly-D-lysine (Sigma) treated T75 culture flask (Celltreat, Pepperell, MA). Cells were cultured in astrocyte media (1\% penicillin/ streptomycin, $10 \%$ horse serum [heat inactivated], within Dulbecco's Minimum Essential Medium (DMEM)) purchased from Invitrogen) until confluence was reached (approximately 2 weeks) with media exchange occurring every 3 to 4 days. Astrocyte culture purity were measured to be $98 \%$ pure using GFAP immunocytochemical staining and DAPI staining (as specified in later sections describing immunohistochemistry procedures).

2.3.2. Astrocyte Culture on Electrospun Fibers and Films-PLLA films and fibers sterilized in 70\% ethanol were placed in a Harrick plasma cleaner (Ithaca, NY) for 90 s. Previous studies have shown strong astrocyte adhesion to fibronectin-coated fibers, ${ }^{16}$ so the surfaces were treated with a $10 \mu \mathrm{g} / \mathrm{mL}$ solution of fibronectin in phosphate buffered saline (PBS; both from Sigma) to encourage astrocyte attachment. The surfaces were dried overnight. Astrocytes were removed from the culture surface with TrypLE heated to $37{ }^{\circ} \mathrm{C}$ and cultured at a density of 66 cells $/ \mathrm{mm}^{2}$. The low density was used in order to observe how an individual cell responded to the surface-so only individual cells were used for morphological analysis.

2.3.3. Immunocytochemistry-To determine the initial astrocyte response to fibers, astrocytes were cultured for different durations. Specifically, astrocytes were cultured for $2 \mathrm{~h}$ to examine initial astrocyte behavior to the surfaces. Later time points $(6,24$, or $48 \mathrm{~h})$ were used to evaluate longer term astrocyte response to fibrous and film surfaces. At each time point, cell fixation occurred over 15 min with $4 \%(\mathrm{v} / \mathrm{v})$ paraformaldehyde mixed with PBS $\left(37^{\circ} \mathrm{C}\right.$; Electron Microscopy Sciences, Hatfield, PA), then permeabilized/blocked for $1 \mathrm{~h}$ (RT) with $0.4 \%(\mathrm{v} / \mathrm{v})$ Triton X-100, 5\% (wt/vol) bovine serum albumin (BSA), dissolved within PBS (Sigma). Incubation of cells occurred overnight at $4{ }^{\circ} \mathrm{C}$ overnight using different primary antibodies (Table 1) dissolved within a solution of $0.1 \%$ Tween-20 and $0.5 \%$ BSA dissolved in PBS. Cells were rinsed with a $0.1 \%(\mathrm{v} / \mathrm{v})$ Tween-20 solution dissolved in a PBS buffer, then introduced to a secondary antibody for a period of $1 \mathrm{~h}$ (RT; Table 1). Solutions containing secondary antibodies were prepared in a buffer of $0.1 \%$ (v/v) Tween-20 and $0.5 \%$ (wt/vol) BSA, all dissolved in PBS. Cells were immersed into PBS and subsequently incubated at RT for 15 min in DAPI (1:1000 dilution; Sigma) in a PBS solution. Finally, samples were washed one last time in PBS $(3 \times 5 \mathrm{~min})$ before imaging. Fluorescent images were taken with $40 \times$ and $20 \times$ magnification on an inverted Olympus IX-81 confocal microscope (Olympus, Tokyo, Japan). Subsequent images generated by the microscope were processed using FIJI and Photoshop software (version CS5, Adobe, San Jose, CA).

2.3.4. Evaluation of Astrocyte Extension-The astrocyte aspect ratio was evaluated using FIJI software to determine if fiber diameter influenced the ability of the astrocyte to elongate along the fibers. At least 39 astrocytes were analyzed from three scaffolds fabricated independently and three astrocyte cultures prepared independently $(N=3)$. Images of cells were analyzed via thresholding the images against lighter objects to 
demarcate the astrocyte perimeter. Cell dimensions were determined using FIJI software. An aspect ratio was used to define the directional extension of an astrocyte and was calculated by taking the longest cellular feature and dividing it by the shortest cellular feature. A ratio equal to 1 depicts a cell with a circular shape, and a ratio more than 1 depicts a cell extending in a particular direction. The DAPI channel was overlaid during analysis to visualize single astrocytes so as to separate them from astrocytes cultured in clumps.

\subsection{Western Blot Analysis}

2.4.1. The Culture and Subsequent Lysis of Astrocytes-Western blot analyses were used to determine if the protein expression from astrocytes varied when cultured on different surfaces. The Western blot procedure was prepared using previously published methods. ${ }^{17}$ For Western blot analysis, astrocyte seeding transpired at higher cell densities (666 cells $/ \mathrm{mm}^{2}$ ). Cells from coverslips (4 in total) were combined to form unique data points as to achieve protein concentrations needed to accurately run the Western blot. Cortical astrocytes were seeded on small diameter fibers, large diameter fibers, or film surfaces for either 1 or 4 days. After culture, samples were rinsed ( 3 times) at $37^{\circ} \mathrm{C}$ with PBS to eliminate any remaining media. The cells were lysed over a period of $5 \mathrm{~min}$ on ice. The lysis buffer was composed of RIPA cell lysis buffer 3 (Enzo Life Sciences, Farmingdale, NY) that contained both enzyme and phosphatase inhibitors (complete ULTRA and PhosSTOP, respectively (both from Roche, Branford, CT)). Each sample was further dissociated using a needle (28-gauge) (Becton Dickenson) before measuring the protein concentration with a BCA assay (ThermoFisher Scientific, Waltham, MA) using guidelines provided by the manufacturer. Laememli buffer (with a $4 \times$ concentration) consisting of $\beta$-mercaptoethanol (SPB; RPI Research Products, Mt. Prospect, IL) was applied to each sample to create a $1 \times$ SPB concentration after which samples were flash frozen and stored at $-80 \mathrm{C}$.

2.4.2. Western Blot Procedure-SDS-PAGE running gels (4\%/10\%) were placed in cassettes ( $1 \mathrm{~mm}$, single use; BioRad, Hercules, CA) using guidelines provided by Abcam. ${ }^{38}$ Samples were thawed and heated using a heat block set to $100^{\circ} \mathrm{C}$ (over 2 min). The samples were then centrifuged at $14000 \mathrm{rpm}(2 \mathrm{~min})$. For each well, $2 \mu \mathrm{g}$ of protein from the lysates was loaded into the gel in $20 \mu \mathrm{L}$ volumes. In other wells, protein standards (BioRad) were run to compare proteins from lysates to proteins of known molecular weight provided by the standard. Proteins were allowed to migrate through each gel for a period of 60 min using a running voltage of $150 \mathrm{~V}$ and a $1 \mathrm{~L}$ volume of running buffer containing Tris-Base $(0.025$ M), glycine (0.0192 M), and SDS (0.1 wt \%/vol).

For the transfer, gels were separated from the holding cassettes, washed with Milli-Q water, then loaded into the transfer cassette with Immun-blot PVFD membranes (BioRad). The transfer was run using a 0.2 A current over $90 \mathrm{~min}$ in a buffer used for transferring $(25 \mathrm{mM}$ Tris-base, $192 \mathrm{mM}$ glycine, 20\% methanol) while on ice.

2.4.3. Western Blot Immunostaining and Imaging-Antibodies used for the Western blot immunostaining procedure are listed in the following table (Table 2). Following transfer, the cassettes were disassembled. The PVFD membranes were placed in a blocking buffer for $1 \mathrm{~h}$ at RT on a shaker. The blocking buffer consisted of $1 \%(\mathrm{v} / \mathrm{v})$ Tween-20 
(Sigma), $20 \mathrm{mM}$ Tris, and $150 \mathrm{mM} \mathrm{NaCl}$ (ThermoFisher), and 5\% (wt/vol) instant nonfat dried milk was added and the $\mathrm{pH}$ equilibrated to 7.6. The primary antibody was placed in the blocking buffer, and the buffer was placed on the membrane overnight (RT) with gentle shaking, followed by $3 \times 5$ min washes with blocking buffer. The membrane was then incubated with secondary antibody (1:10 000 dilution in blocking buffer) for $2 \mathrm{~h}$ on a shaker, then washed $3 \times 5 \mathrm{~min}$ in blocking buffer. A Western blotting detection reagent (Amersham ECL; GE Healthcare, Chicago, IL) was placed on the membrane for 2 min before imaging. Imaging of the membrane occurred using a ChemiDoc XRS imaging system and ImageLab Software (BioRad). The molecular weight standard was imaged immediately after.

Stripping of membranes occurred using $2 \times 10$ min incubations in a buffer consisting of 200 $\mathrm{mM}$ glycine, $3.5 \mathrm{mM} \mathrm{SDS}$, and $1 \%(\mathrm{v} / \mathrm{v})$ Tween-20 (pH 2.2), then washed $3 \times 5 \mathrm{~min}$ in a PBS solution and then subsequently washed $2 \times 5$ min using blocking buffer. The immunostaining procedure was then repeated on each membrane to probe for the actin control.

FIJI software was used for blot analysis. For all experiments, cells were cultured on flat surfaces using tissue culture plastic (TCP), and this group of cells served as a control. Each group consists of at least five independently produced scaffold replicates and five separate astrocyte isolations. Western blot images displayed in subsequent figures are from one representative blot. The resulting graphs representing the data from these blots are averages of five separate Western blot experiments.

\subsection{Astrocyte-Neuron Coculture}

2.5.1. Neuron Dissociation-For assessing the ability of astrocytes on different diameter fiber scaffolds to direct neurite extension and protect neurons from glutamate excitotoxicty, we selected dissociated neurons from dorsal root ganglia (DRG). Although DRG cell bodies reside in the peripheral nervous system, their axons project into the central nervous system. ${ }^{39}$ As such, they are an appropriate cell source to examine astrocyte-induced neurite outgrowth. Primary neurons were placed on astrocytes cultured on fibrous scaffolds to determine if fiber diameter correlated with neurite guidance, outgrowth, and neuron protection in the coculture. DRG were excised from P2 rats (Sprague-Dawley); then primary neurons were dissociated from the DRG using a modified process described by Koppes et al.

40 The DRG tissue was enzymatically digested for a period of $50 \mathrm{~min}$ at $37{ }^{\circ} \mathrm{C}$ using a buffer consisting of $0.1 \%$ (wt/vol) Trypsin (Corning, Corning, NY) and $1 \mathrm{mg} / \mathrm{mL}$ collagenase A (Sigma) dissolved in PBS set to a pH of 7.4 in a tissue culture incubator $\left(5 \% \mathrm{CO}_{2}\right)$. The DRG were resuspended every 10 min during the incubation to improve the collagenase digestion. The collagenase digestion was followed by a 10 min incubation in $0.25 \%$ Trypsin, PBS (pH 7.4). Cells were pelleted by centrifugation for a buffer exchange into astrocyte media. The pellet was resuspended by trituration (fewer than 10 times) with a flamepolished Pasteur pipet.

\subsubsection{Dissociated Neuron-Astrocyte Coculture on Fiber Scaffolds-The} electrospun fibers and films were sterilized in ethanol, plasma treated for $90 \mathrm{~s}$, then incubated with a solution of $10 \mu \mathrm{g} / \mathrm{mL}$ of fibronectin (Sigma) in HBSS until dry. Astrocytes 
were seeded at different times to achieve sets with both 1-day-in-culture and 4-daysinculture at the time the neurons were seeded (see Figure 3I for timeline). Therefore, the times ( 1 day and 4 day) represent the age of the astrocytes in culture. All neurons were cultured for the same amount of time ( 1 day). The astrocytes were cultured on the surfaces at 666 cells $/ \mathrm{mm}^{2}$ to completely cover the film or fiber scaffolds with astrocytes, while the isolated neurons were cultured at 133 cells $/ \mathrm{mm}^{2}$. The astrocyte seeding density was selected to ensure that extending neurites were extending over astrocytes and not fibers beneath the astrocytes. The neurons were cultured at a low density in order to reduce overlap between neighboring neurons. After seeding, the scaffolds were gently rocked to distribute the neurons. The neuron/astrocyte coculture was incubated for $24 \mathrm{~h}$ in astrocyte media supplemented with $25 \mathrm{ng} / \mathrm{mL}$ NGF (Invitrogen).

\subsubsection{Astrocyte-Neuron Coculture Fixing and Immunocytochemistry-After} $24 \mathrm{~h}$, the scaffolds were washed over $15 \mathrm{~min}$ in a warmed $4 \%$ paraformaldehyde fixative, then rinsed (three times, $5 \mathrm{~min}$ for each rinse) in ice-chilled PBS. Cells cultured on scaffolds were blocked and permeabilized for 15 min with a $0.01 \%$ (v/v) Triton-X 100 (Sigma), 5\% $(\mathrm{wt} / \mathrm{vol}) \mathrm{BSA}$, and saline buffered with tris and maintained at a $\mathrm{pH}$ of 7.4 (Tris-buffered saline (TBS), ThermoFisher), then replaced with a solution containing primary antibodies (for at least $12 \mathrm{~h}$ at $4{ }^{\circ} \mathrm{C}$ ). The solution containing primary antibodies was comprised of $0.1 \%(\mathrm{v} / \mathrm{v})$ Tween-20 (P1379, Sigma-Aldrich), 5\% (wt/vol) BSA, and Tris-buffered saline maintained at a pH of 7.4 with anti-GFAP antibody (1:800 dilution) and RT-97-S antibody (1:400 dilution) for neurofilament ( $64 \mu \mathrm{g} / \mathrm{mL}$, Developmental Studies Hybridoma Bank (DSHB) at the University of Iowa, Iowa City, IA). Scaffolds were washed $3 \times 5 \mathrm{~min}$ in a solution consisting of TBS and $0.1 \%(\mathrm{v} / \mathrm{v})$ Tween-20, before incubation in the secondary antibody solution: donkey antimouse AlexaFlour 594 ( $2 \mathrm{mg} / \mathrm{mL}$, Life Technologies) antibody, goat antirabbit (AlexaFlour 488, Life Technologies) antibody, and DAPI (1 $\mathrm{mg} / \mathrm{mL}$, ThermoFisher) were all placed in a solution consisting of TBS, $0.1 \%(\mathrm{v} / \mathrm{v})$ Tween 20, and 5\% (wt/vol) BSA at a 1:1000 dilution. The secondary antibody was incubated at room temperature for $1 \mathrm{~h}$, then washed (three times, 5 min each wash) in PBS, before imaging.

2.5.4. Isolated Neuron Analysis-A spinning disk confocal microscope (Olympus IX-81) was used to capture images of astrocytes and neurons. Approximately 10 neurons were imaged for each scaffold. Images were processed with a maximum intensity $z$ projection, followed by background subtraction (rolling ball $=50)$ using FIJI software $(\mathrm{NIH}$, Bethesda, MD). If necessary, the MosaicJ plugin from FIJI was used to assemble neuron images.

After 1 day or 4 days of astrocyte culture, neurite outgrowth on astrocyte-covered films and neurite outgrowth on small diameter fibers and large diameter fibers was quantified by manually tracing neurites using Neurolucida 10.0 software (MBF Biolabs, Williston, VT). Matlab software (Mathworks, Natick, MA) was used to calculate the longest neurites, the amount of primary neurites, total outgrowth from each branch point, and the number of branch points per neuron. The single longest neurite measured from initial outgrowth from the soma to its tip was termed as being the longest neurite. Neurites were termed as being 
primary neurites if their outgrowth originated from the soma. The "mean neurite length parallel to fiber orientation" was determined by calculating the mean length of all neurites that oriented along the $90^{\circ}$ and $270^{\circ}$ axis (parallel to fiber orientation). At least three independent astrocyte and neuron isolations were used to generate data and graphs along with four independently produced fiber scaffolds.

2.5.5. LDH Assay-Since astrocytes on fibers increase their expression of GLT-1 and uptake of extracellular glutamate, we wanted to study if astrocytes on fibers were capable of rescuing neurons from glutamate concentrations known to induce neuronal excitotoxicity. The dissociated neuron-astrocyte cocultures were prepared in a manner described previously in section 2.5.2. The medium was changed $1 \mathrm{~h}$ before the assay began to remove the effects of any cells that died during the plating process. A solution of $50 \mathrm{mM} \mathrm{L-glutamic}$ acid (G1251, Sigma) in a PBS solution maintained to a $\mathrm{pH}$ of 7.4 was placed into each experimental well to achieve a $20 \mathrm{mM}$ concentration, then cocultures were incubated for 24 h. ${ }^{41}$ After $24 \mathrm{~h}, 100 \mu \mathrm{L}$ of media was removed from each well, and the sample was quickly cooled using dry ice. The frozen samples were then transferred to a freezer $\left(-80^{\circ} \mathrm{C}\right)$ until used in the LDH assay. To estimate the maximum population LDH release, untreated samples were lysed with RIPA buffer and flash frozen.

All samples were thawed in a water bath maintained at a temperature of $37^{\circ} \mathrm{C}$, centrifuged for $15 \mathrm{~min}$ at $14000 \mathrm{rpm}$ at $4{ }^{\circ} \mathrm{C}$, then analyzed using an LDH assay kit (MAK066, Sigma). The "\% survival after excitotoxicity" was determined by dividing the amount of LDH released in the treated sample by the total amount of LDH released from the untreated lysed samples. The results represent three independently fabricated fiber scaffolds, three independently isolated astrocyte cultures, and three independently isolated neuron cultures.

\subsection{Summary of Statistical Analysis}

Sigma Plot software (version 11.0, Systat Software Inc., San Jose, CA) was used for statistical analysis. The standard deviations from initial results were used to calculate the sample size for each experiment to achieve a power of 0.9. Significance between groups was determined using a one way ANOVA with the exception of fiber density. A KolmogorovSmirnov test was used to test all groups for normality. For normally distributed data, an ANOVA test was conducted, and a Tukey HSD post hoc analysis test was included. For nonnormal distributions, a nonparametric ANOVA was conducted on ranks. The groups used to evaluate fiber density had unequal variances and required a different test (Mann-Whitney). $p$ values $\leq 0.05$ indicate statistically significant differences between groups. Data reported in the text are presented as the mean \pm standard error. Differences between groups were considered significant when $p<0.05$. All data were constructed using at least three independently fabricated fiber batches and three separate cultures of astrocytes and/or neurons, unless specified otherwise. All graphs show the mean \pm standard error. Fiber alignment was compared using a Brown-Forsythe test using JMP software (SAS, Cary, NC). 


\section{RESULTS}

\subsection{Electrospun Fibers Prepared with Different Diameters}

The study's purpose was to create scaffolds with unique fiber diameters while introducing as few confounding variables as possible. The solvent in the electrospinning solution was identified as one potential source of error. ${ }^{18}$ The use of different solvents to create the different diameters may introduce changes in fiber crystallinity or impact the extent of solvent retained within the fibers. Therefore, it was important that the solvent be consistent for all experimental groups. HFIP was selected as a solvent for this study because preliminary tests showed that HFIP could accommodate a range of polymer concentrations without the electrospinning jet collapsing into electrospraying (Figure S1A). Figure S1A shows that increasing the PLLA concentration from $6 \%$ to $12 \%$ initiated a linear increase of the fiber diameter ranging from 363 to $808 \mathrm{~nm}$.

We generated film surfaces to serve as nontopographical controls (Figure 1A). The 7\% and $12 \%$ PLLA solutions produced fibers with significantly different diameters between 0 and 1 $\mu \mathrm{m}$, so these were chosen as the small diameter (Figure 1B) and large diameter fibers (Figure 1C) for the study. The average diameter of the large diameter fibers was $808 \pm 44$ $\mathrm{nm}$, while the average diameter of the small diameter was $386 \pm 18 \mathrm{~nm}$ (Figure 1D). These diameters were also selected to simulate increased axon diameters as neurons mature. While axons can vary from nanometers to tens of microns in diameter, it has been suggested that the average mature axon in the body is approximately $1 \mu \mathrm{m}$ in diameter. ${ }^{25,26}$ The electrospinning parameters were adjusted to control the fiber alignment to isolate the fiber diameter as the most prominent variable to affect the astrocyte response.

The fiber diameter decreased as the PLLA concentration decreased (Figure S1A), but the fibers also became significantly less aligned (Figure S2G). To improve the small diameter fiber alignment, the collection mandrel collection speed was doubled to $2000 \mathrm{rpm}$ (Table 3). The increased rotation speed improved the alignment but reduced the collection efficiency of the fibers. The aim was to achieve a monolayer of fibers with both the small and large diameter fiber scaffolds with similar coverage of fibers. To achieve this, the collection time was increased from 10 to $15 \mathrm{~min}$ for the small fibers. The resulting average density of the large fibers was $857 \pm 32$ fibers $/ \mathrm{mm}$ for the large fibers and $2052 \pm 79$ fibers $/ \mathrm{mm}$ for the small fibers (Figure 1E). The coverage of the fibers was estimated by multiplying the fiber density by the fiber diameter (diameter density product). The fiber coverage was 69.2 $\pm 13.9 \%$ fibers $/ \mathrm{mm}$ for the large fibers and $79.2 \pm 14.7 \%$ for the small fibers (Figure 1F). The longer collection time resulted in similar fiber coverage between the large and small diameter scaffolds, with no statistically significant differences. The pump rate and chamber humidity were also adjusted to improve the fiber formation and collection (Table 3 ). This troubleshooting resulted in two electrospun fiber scaffolds with distinct fiber diameters (Figure 1D) but similar fiber alignment (Figure 1F, G, H), coverage (Figure 1F), and hydrophilicity (Figure S3). 


\subsection{Astrocyte Elongation on Aligned Fibers of Different Diameter}

To determine the astrocyte response to the controlled surface topographies, primary rat astrocytes isolated from the cortex were placed on small and large diameter fiber surfaces, with a flat PLLA film as the control surface. Preliminary experiments suggested that astrocytes adhered to the surfaces within $2 \mathrm{~h}$ and reached an elongated morphology by $24 \mathrm{~h}$ (Figure S4), which would continue for all time points examined (4 days).

The aspect ratio of the astrocyte cell body is defined as the ratio of the longest cell aspect and the shortest cell aspect and provides a measure of how elongated the astrocyte becomes. The results showed that within $2 \mathrm{~h}$ astrocytes became significantly more elongated on the large fibers than on film or small fibers. Astrocytes on the large fibers remained significantly more elongated for the remainder of the time tested (Figure $2 \mathrm{~A}$ ). The astrocyte elongation was greatest after $24 \mathrm{~h}$, with aspect ratios of $7.8 \pm 0.6$ on large fibers, $4.4 \pm 0.4$ on small fibers, and $2.4 \pm 0.3$ on film surfaces. Astrocytes cultured on the small fibers only became significantly more elongated than those on the film after $96 \mathrm{~h}$ in culture (Figure 2A). Representative images from this study reveal that after 1 day in culture, astrocytes cultured on films (Figure 2B) were rounded in shape. Astrocytes cultured on small diameter fibers (Figure 2C) started to recognize fiber topography and orient along the fibers, and this orientation was more pronounced when astrocytes were cultured on large diameter fibers (Figure 2D). Other representative images of astrocytes cultured for 4 days on the various surfaces reveal astrocytes continuing to extend in all directions on films (Figure 2E). Astrocyte extension along small diameter fibers (Figure 2F) was more elongated than after 1 day but still not as elongated as astrocytes cultured on large diameter fibers (Figure 2G).

For all further experiments, the 1 day time point was selected because it was the earliest time where the astrocyte morphology on the small fibers separated from those on the film substrates. The 4 day time point was chosen because it was the first time point where the astrocyte morphologies were statistically different on all surfaces. Four days is also a time point that is commonly used in other studies, allowing for our findings to be compared with other observations found in the literature.

\subsection{The Effect of Fiber Diameter on Astrocyte Mediated Neurite Guidance}

Astrocytes have shown an ability to use cues provided by the substrate topography to guide neurites extending from neurons in culture. ${ }^{42}$ Because astrocytes were observed to elongate more extensively on larger diameter fibers, we hypothesized that increasing the fiber diameter would enhance astrocyte-mediated neurite guidance. To prove or disprove this hypothesis, primary astrocytes isolated from the cortex were cultured on each surface for either 1 day (1D-astrocyte) or 4 days (4D-astrocyte) before neurons were introduced. Dissociated primary DRG neurons were seeded on all surfaces at the same time and were cultured on all surfaces for the same amount of time ( $24 \mathrm{~h})$. See Figure $3 \mathrm{I}$ for the timeline. The neurite arbors were traced to measure differences in neurite outgrowth.

Representative images from these experiments show astrocyte-neuron coculture results. After 1 day of culture (proceeded by 1 day of astrocyte culture on the surfaces), neurites extended in all directions when astrocytes were cultured on films (Figure 3A). Neurites 
extending on astrocytes cultured on small diameter fibers (Figure 3B) or large diameter fibers (Figure 3C) extended along the astrocytes. The same general findings were observed when astrocytes were cultured on the different surfaces for 4 days. Neurites extended in all directions when astrocytes were cultured on films (Figure 3D). Neurite extension, again, followed the orientation of the aligned astrocytes on small diameter (Figure 3E) or large diameter fibers (Figure 3F). Neurite images from these experiments were traced. From the 1 day astrocyte culture experiments, neurite outgrowth was slightly longer on large diameter fibers than what was observed on small diameter fibers (Figure 3G). From the 4 day astrocyte culture experiments, neurite outgrowth was shorter overall than the 1 day astrocyte culture experiments, and there were no noticeable differences in neurite outgrowth between the two fiber types (Figure 3H).

The tracing led to two important observations: First, the diameter of the fibers affected astrocyte-mediated neurite outgrowth along the aligned fibers. At both time points, the mean neurite length along the fiber orientation increased as the fiber diameter increased (Figure $3 \mathrm{~J})$. This confirms the hypothesis that the more elongated astrocyte morphology on large diameter fibers is associated with increased neurite outgrowth. Second, neurites in the cocultures were significantly less branched on fiber surfaces, when compared to those on film surfaces. For both time points measured, the neurites on the large fibers had significantly fewer branches per length than those on the film substrates. Astrocytes on the small fibers took longer to facilitate significantly decreased neurite branching, when compared to astrocytes placed on large fibers. The neurites extending along astrocytes cultured on small diameter fibers only showed significantly less branching than those on the film control on the 4-day astrocytes and were not significantly different from those on the large fibers (Figure $3 \mathrm{~K}$ ). The decreased branching was primarily due to fewer branch points and not to differences in length (Figure S6C), since statistical differences were not observed between the longest neurite (Figure S6A) or the total length (Figure S6B) in cocultured neurites on any substrate.

\subsection{Fiber-Mediated Astrocyte Neuroprotection}

Experiments were performed to test whether the astrocyte expression of glutamate transporters (GLT-1 and GLAST) was dependent on fiber diameter. Previous studies report that electrospun fibers increase astrocyte production of GLT-1, which increased their capacity to remove glutamate from the extracellular environment. ${ }^{16}$ In this study, astrocytes interacted with each surface for 1 day or 4 days then were lysed to measure GLT-1 and GLAST expression using Western blot (Figure 4A-G).

GLT-1 was significantly increased on both fiber substrates but was not sensitive to fiber diameter (Figure 4H). After 1 day in culture, there were no differences in GLT-1 in any of the experimental groups tested. After 4 days, astrocyte GLT-1 expression was increased on both small diameter and large diameter fiber surfaces compared to the film. But, this nearly 3-fold increase was similar in both the small and large fiber 4Dastrocyte cultures. Therefore, the hypothesis was rejected that increases in GLT-1 are related to fiber diameter. GLAST is the other major astrocyte glutamate transporter and was studied alongside GLT-1. After 4 
days in culture, GLAST expression on the large and small fibers was not statistically different from the film control (Figure 4I)

To test whether the increase in GLT-1 resulted in increased neuroprotection, the same coculture protocol was used as in section 3.3, but the cocultures were exposed to a high glutamate solution for $24 \mathrm{~h}$. The common method to model excitotoxicity in vitro is to treat the cultures with $1 \mathrm{mM}$ glutamate for $10 \mathrm{~min}^{43}$ or $30 \mathrm{~min}^{44,45}$ and to measure cell death with an LDH assay. Cells release LDH following plasma membrane damage, and this approach is a quantitative method to determine cell death. However, one report showed that the differences in released astrocyte LDH was most distinguishable $24 \mathrm{~h}$ after exposure to glutamate. ${ }^{46}$ Furthermore, these prior studies did not report correcting for the $\mathrm{pH}$ of the glutamate solution.

Our experiments showed that glutamate lowered the $\mathrm{pH}$ of the media, and Croce and colleagues reported that the acidity of the environment during glutamate exposure was a confounding variable. ${ }^{41}$ Astrocyte glutamate metabolism is sensitive to $\mathrm{pH}$. Acidity is reported to inhibit the glutamine synthetase pathway, which increases glutamate accumulation in the extracellular environment, compounds the excitotoxic effects of glutamate, and leads to a higher degree of cell death. ${ }^{47}$ Croce et al. found that the cell death -as measured by LDH - was no longer observed after correcting the $\mathrm{pH}$ of the $2 \mathrm{mM}, 5$ $\mathrm{mM}$, and $10 \mathrm{mM}$ glutamate solutions. After correcting the $\mathrm{pH}$, only the $20 \mathrm{mM}$ glutamate solution treatment significantly decreased cell viability (after $24 \mathrm{~h}$ ) compared to the untreated control. ${ }^{41}$ Informed by these previous results, the cocultures in our study were treated with $20 \mathrm{mM}$ glutamate with a pH of 7.4 for $24 \mathrm{~h}$. An LDH assay was then performed on the media to measure cell death.

Following these initial observations, we conducted an LDH assay as specified in Figure 5A. The LDH measurements were normalized to the maximum LDH released when untreated cocultures from each surface were lysed. Therefore, the results are reported as a percentage of the maximum LDH released. The survival for the 1D-astrocyte cocultures was similar on all surfaces (65-68\% survival). Glutamate treatment of the 4D-astrocyte cocultures resulted in a $38.3 \pm 6.8 \%$ survival on films after glutamate treatment. Importantly, the survival on both the small and large fiber substrates significantly increased to $77.6 \pm 1.4 \%$ and 73.9 $\pm 3.0 \%$, respectively (Figure 5B). For context, neuron-only controls resulted in $43-48 \%$ survival (Figure S8A), while astrocyte-only controls showed an approximately $87 \%$ survival (Figure S8B). However, it is important to consider that these may not be ideal controls because interactions between neurons and astrocytes are known to alter glutamate transporter clustering, which affects glutamate transporter activity. ${ }^{48}$ So, the controls cannot account for the synergistic effect of the neuron-astrocyte interactions in coculture. The coculture excitotoxicity data correspond with the increases in GLT-1 in the 4D-astrocyte Western blot date (Figure $4 \mathrm{H}$ ). As a result, the hypothesis was upheld that increases in astrocyte GLT-1 on fiber substrates result in improved neuron survival.

The cytoskeletal marker GFAP was also measured because it is a common marker for reactivity-sizable GFAP expression increases are associated with astrocyte reactivity. ${ }^{1}$ Analysis of GFAP expression was conducted using Western blot analysis to determine if 
morphological changes observed in astrocytes on the different diameter fibers correlated with GFAP production (Figure S7). While GFAP intensity increased in astrocytes on the film substrates after 4 days and decreased on fibrous surfaces, there were no statistically significant changes in astrocyte GFAP on any surface for all the time points measured (Figure S7).

\section{DISCUSSION}

\subsection{Astrocyte Elongation}

The ability of the electrospun fiber diameter to influence astrocyte elongation, astrocytemediated neurite guidance, and astrocyte-mediated neuroprotection (Figure 6) was investigated here. The results showed that increased fiber diameters within aligned fiber surfaces significantly increased astrocyte elongation. Astrocytes on the large fiber surfaces were $1.8 \times$ more elongated than those on small fibers and $3.3 \times$ more elongated than those on film substrates after $24 \mathrm{~h}$. This significantly more elongated astrocyte morphology was observed on large diameter fibers as soon as $2 \mathrm{~h}$ following seeding. The results show a significant, diameter-dependent change in astrocyte morphology for up to 4 days.

$\mathrm{Qu}$ et al. tested the astrocyte response to unaligned electrospun silk fibroin fibers with similar diameters and found that the larger diameters restricted astrocyte spreading. ${ }^{30}$ These seemingly contradictory findings fit with ours based on the theory that the larger diameter fibers present a more imposing barrier. The unaligned larger diameter fibers inhibited astrocyte spreading because the unaligned fibers did not create channels between individual fibers. ${ }^{30}$ When the fibers are aligned, the larger channels created by larger fibers improve astrocyte elongation (Figure 2). This theory was proposed by Biran and colleagues, who found that astrocyte orientation increased as the roughness of the underlying aligned topography of stamped polystyrene surfaces was increased from 45 to $492 \mathrm{~nm} .{ }^{42}$ The results reported here follow those of Biran et al., by showing that larger topographical features$400-800 \mathrm{~nm}$ in diameter-increase the directed elongation of astrocytes (Figure 2A). However, these data cannot be directly compared because the materials, preparation techniques, and topographical feature sizes explored are different.

\subsection{Neurite Guidance}

Neurons cocultured with astrocytes on aligned fibers were more aligned and less branched than those on film substrates (Figure 3G, H), and neurite extension along the aligned fibers was parallel to both the fiber orientation and the astrocyte elongation. The neurite growth length parallel to fiber alignment was dependent on fiber diameter and increased as fiber diameter increased (Figure 3J). This supports previous findings that showed that the directed alignment and elongation of astrocytes improved directional neurite outgrowth. ${ }^{27,42,49}$ Aligned astrocytes produce aligned arrays of adhesive ligands (laminin, fibronectin, and neural cell adhesion molecule (NCAM)) along with inhibitory boundaries created by chondroitin sulfate proteoglycans (CSPGs). ${ }^{42}$ These channels of ligands facilitate directional neurite growth parallel to the underlying aligned surface topography ${ }^{42}$ by aligning the cytoskeleton of migrating neurites.50,51 
Neurite tracing results revealed that the alignment of the fibers did not increase the neurite length. The longest neurites (in any direction) were not statistically different to those observed on films, small diameter fibers, or large diameter fiber substrates (Figure S6A). Furthermore, statistical differences were not observed in the total neurite length when comparing neurite outgrowth on the different surfaces (Figure S6B). Therefore, the directional alignment of the fibers ensured that the longest neurites were in the direction of the fiber alignment, and not in a haphazard radial pattern, as on the film (Figure 3G, H). The increased fiber diameter improved the directional guidance of neurite outgrowth without significantly affecting the overall length.

While fiber diameter was able to influence astrocyte-mediated neurite outgrowth at both time points, it was also observed that the astrocyte culture time slightly affected the neurite outgrowth. The directional neurite length on the 1D-astrocytes was consistently longer than the length on the 4D-astrocytes for both fiber substrates, although the differences were not statistically significant (Figure 3J). We previously observed a similar but significant decrease in DRG neurite outgrowth on astrocyte/fiber surfaces as astrocyte culture time was increased (2110 nm diameter fibers). ${ }^{17}$ Interestingly, when the direction of the neurite outgrowth was not considered, the trend was lost-the longest neurite length was shorter on the 1Dastrocytes compared to the 4D-astrocytes on the film and large fiber substrates (Figure S6A). This suggests that, after longer culture times, the astrocytes were not inhibiting neurite growth but rather slightly masking the highly aligned topography, causing less aligned growth.

The neurites cocultured with astrocytes on the fibers had significantly less branching per length than those cultured on the film control, but statistical differences were not observed between the different fiber diameter groups (Figure 3K). However, the astrocyte-mediated decrease in neurite branching required a longer astrocyte culture time on small diameter fibers. In contrast to the results observed on films, branching was decreased significantly on large diameter fibers in both the 1D- and 4D-astrocyte cocultures, while on the small diameter fibers, branching was only significantly decreased in the 4D-astrocyte coculture. These data suggest that changes in fiber diameter affect the rate of astrocyte-mediated neurite branching. Overall, neurons within the coculture decreased branching in favor of directional elongation on astrocyte/fiber surfaces. A similar phenomenon has been observed during cerebral cortex development as neurons migrated along fibers created by extended radial glia (astrocyte precursors). The neurites continuously contacted the radial glia and delayed branching until they reached the outer layer of the cortex. ${ }^{52,53}$ The decreased branching on the astrocyte/electrospun fiber substrates suggests that the aligned fiber environment prioritizes fasciculation and migration over branching and networking. ${ }^{20}$

\subsection{Astrocyte-Mediated Neuroprotection}

Following spinal cord injury (SCI), astrocytes downregulate their production of GLT-1, ${ }^{54}$ and the reduced GLT-1 expression worsens functional and histological outcomes following SCI. ${ }^{55}$ Increasing GLT-1 expression following SCI through the use of viral vectors can reverse neuropathic pain but can also lead to additional neuronal degeneration. ${ }^{56,57}$ Thus, if a biomaterial design could naturally upregulate astrocyte production to GLT-1, it may at 
least in part help explain why fibrous topographies induce axonal regeneration following acute spinal cord injury. ${ }^{31}$

One of the study's goals was to determine if changes in fiber diameter influenced astrocyte production of GLT-1. Thus, we assessed GLT-1 expression in addition to GLAST and GFAP. Astrocyte GLAST, GFAP, and GLT-1 expression were analyzed on each of the fiber diameters. Overall, fiber diameter is not the major variable affecting GLAST, GFAP, or GLT-1 expression. However, the topography provided by the electrospun fibers promoted neuroprotection through increased glutamate transporter expression.

Astrocyte GLAST expression increased as fiber diameter increased (Figure 4I).

Extrapolating this trend would suggest that the increase in GLAST would become significant above a certain fiber diameter threshold. Unfortunately, this trend does not appear to be linear. Zuidema et al. observed a similar expression of GLAST on aligned PLLA fibers with diameters of $2400 \mathrm{~nm}$, which were also not significantly different from the film control. This suggests that GLAST expression may be moderately sensitive to fiber diameter, but fiber diameter is not a major variable affecting GLAST expression.

Increased expression of GFAP is a marker of increased astrocyte reactivity, and large amplifications of GFAP are generally indicative of a reactive astrocyte phenotype. ${ }^{5}$ On both fiber surfaces, GFAP expression decreased slightly compared to the film control but was not sensitive to changes in fiber diameter (Figure S7). Although the decrease was not statistically significant, these results are similar to previous findings that showed a significant decrease in GFAP on $1200 \mathrm{~nm}$ diameter fibrous substrates ${ }^{15}$ and fibrous substrates of unknown diameter. ${ }^{58}$

Astrocyte GLT-1 expression on both the small and large diameter fiber surfaces was increased approximately 3 -fold after 4 days, relative to the film control (Figure $4 \mathrm{H}$ ). This increase in GLT-1 expression was associated with a nearly $40 \%$ increase in cell survival when the cocultures were exposed to high levels of glutamate (Figure 4M). Zuidema et al. found previously that GLT-1 and glutamate uptake are significantly increased on $2400 \mathrm{~nm}$ diameter fibers, ${ }^{16}$ and Lau et al. found that GLT-1associated-mRNA was increased on 400$450 \mathrm{~nm}$ diameter fibers. ${ }^{13}$ Both studies also showed that the fiber alignment was not a major factor affecting GLT-1 expression. The results here support these previous findings but also answer one prominent question not explored in these studies: the fiber diameter is not a major factor in stimulating GLT-1 expression in astrocytes (Figure 4H). Combining these findings shows that there is a large range of fiber diameters (400-2400 nm) and fiber alignments (unaligned-highly aligned) that will elicit a similar and significant GLT-1associated neuroprotective effect in astrocytes.

Inadequate glutamate uptake contributes to the pathology of many CNS disorders (reviewed by Danbolt ${ }^{59}$ ). The results here show that the topography of the physical environment can have a significant effect on astrocyte-mediated glutamate neuroprotection by modifying GLT-1 expression. 


\section{CONCLUSION}

This study's aim was to examine how changes in electrospun fiber diameter affected aspects of astrocyte morphology and phenotype (Figure 6). Doubling (approximately) the aligned electrospun fiber diameter from 386 to $808 \mathrm{~nm}$ caused a significant increase in astrocyte elongation, which also improved directional neurite outgrowth. Both the small and large fiber substrates significantly increased GLT-1 production over the film control, which was associated with a $40 \%$ increase in astrocyte/neuron coculture survival. While the findings suggest that GLT-1 expression is not dependent on fiber diameter, they also suggest that simply engineering the topographical environment can significantly improve neuroprotection in a high glutamate environment. The large diameter fibers had the added benefit of increased directional neurite outgrowth length and a more rapid astrocyte response with increased process elongation. Thus, if aligned, electrospun fibers are utilized for directing axonal regeneration following central nervous system injury, it would be best to select fibers with larger diameters capable of triggering quick morphological changes in astrocytes to better direct the extension of axons.

\section{Supplementary Material}

Refer to Web version on PubMed Central for supplementary material.

\section{ACKNOWLEDGMENTS}

Most importantly, the authors wish to acknowledge Dr. Alexander Mongin for his expertise in troubleshooting the astrocyte culture and western blot. We also wish to acknowledge the support provided by Anthony D'Amato, Alexis Ziemba, Manoj Gottipatti, Rachael Melita, Anders Jepson, Tim Piette, Ray Dove, and Deniz Rende.

Funding

This work was supported by the New York State Spinal Cord Injury Research Board Predoctoral Fellowship Award [contract number C30606GG]; National Science Foundation [grant number 1105125]; and the National Institutes of Health [grant number NS092754].

\section{ABBREVIATIONS}

$\begin{array}{ll}\text { DRG } & \text { dorsal root ganglia } \\ \text { SEM } & \text { scanning electron microscopy } \\ \text { HFP } & 1,1,1,3,3,3 \text {-Hexafluoro-2-propanol } \\ \text { GFAP } & \text { glial fibrillary acidic protein } \\ \text { GLAST } & \text { excitatory amino acid transporters 1 } \\ \text { GLT-1 } & \text { excitatory amino acid transporters 2 } \\ \text { ECM } & \text { extracellular matrix } \\ \text { CNS } & \text { central nervous system } \\ \text { PLLA } & \text { poly-L-lactic acid }\end{array}$




\section{REFERENCES}

(1). Sofroniew MV Molecular Dissection of Reactive Astrogliosis and Glial Scar Formation. Trends Neurosci. 2009, 32 (12), 638-647, DOI: 10.1016/j.tins.2009.08.002. [PubMed: 19782411]

(2). White RE; Jakeman LB Don't Fence Me in: Harnessing the Beneficial Roles of Astrocytes for Spinal Cord Repair. Restor. Neurol. Neurosci 2008, 26 (2-3), 197-214. [PubMed: 18820411]

(3). Hamby ME; Sofroniew MV Reactive Astrocytes as Therapeutic Targets for CNS Disorders. Neurotherapeutics 2010, 7 (4), 494-506. [PubMed: 20880511]

(4). Allen NJ; Barres BA Neuroscience: Glia — More than Just Brain Glue. Nature 2009, 457 (7230), 675-677. [PubMed: 19194443]

(5). Sofroniew MV; Vinters HV Astrocytes: Biology and Pathology. Acta Neuropathol. 2010, 119 (1), 7-35. [PubMed: 20012068]

(6). Buss A; Brook GA; Kakulas B; Martin D; Franzen R; Schoenen J; Noth J; Schmitt AB Gradual Loss of Myelin and Formation of an Astrocytic Scar during Wallerian Degeneration in the Human Spinal Cord. Brain 2004, 127 (1), 34-44. [PubMed: 14534158]

(7). Ridet JL; Privat A; Malhotra SK; Gage FH Reactive Astrocytes: Cellular and Molecular Cues to Biological Function. Trends Neurosci. 1997, 20 (12), 570-577. [PubMed: 9416670]

(8). Hara M; Kobayakawa K; Ohkawa Y; Kumamaru H; Yokota K; Saito T; Kijima K; Yoshizaki S; Harimaya K; Nakashima Y; Okada S Interaction of Reactive Astrocytes with Type I Collagen Induces Astrocytic Scar Formation through the Integrin-N-Cadherin Pathway after Spinal Cord Injury. Nat. Med 2017, 23 (7), 818-828. [PubMed: 28628111]

(9). Mattotti M; Alvarez Z; Ortega JA; Planell JA; Engel E; Alcántara S Inducing Functional Radial Glia-like Progenitors from Cortical Astrocyte Cultures Using Micropatterned PMMA. Biomaterials 2012, 33 (6), 1759-1770. [PubMed: 22136716]

(10). Cao H; Marcy G; Goh ELK; Wang F; Wang J; Chew SY The Effects of Nanofiber Topography on Astrocyte Behavior and Gene Silencing Efficiency. Macromol. Biosci 2012, 12 (5), 666-674. [PubMed: 22411782]

(11). Chow WN; Simpson DG; Bigbee JW; Colello RJ Evaluating Neuronal and Glial Growth on Electrospun Polarized Matrices: Bridging the Gap in Percussive Spinal Cord Injuries. Neuron Glia Biol. 2007, 3 (2), 119-126. [PubMed: 18458759]

(12). Delgado-Rivera R; Harris SL; Ahmed I; Babu AN; Patel RP; Ayres V; Flowers D; Meiners S Increased FGF-2 Secretion and Ability to Support Neurite Outgrowth by Astrocytes Cultured on Polyamide Nanofibrillar Matrices. Matrix Biol. 2009, 28 (3), 137-147. [PubMed: 19243701]

(13). Lau CL; Kovacevic M; Tingleff TS; Forsythe JS; Cate HS; Merlo D; Cederfur C; Maclean FL; Parish CL; Horne MK; Nisbet DR; Beart PM 3D Electrospun Scaffolds Promote a Cytotrophic Phenotype of Cultured Primary Astrocytes. J. Neurochem 2014, 130 (2), 215-226. [PubMed: 24588462]

(14). Min SK; Kim SH; Kim CR; Paik S-M; Jung S-M; Shin HS Effect of Topography of an Electrospun Nanofiber on Modulation of Activity of Primary Rat Astrocytes. Neurosci. Lett 2013, 534, 80-84. [PubMed: 23178191]

(15). Puschmann TB; Zanden C; De Pablo Y; Kirchhoff F;. Pekna M; Liu J; Pekny M Bioactive 3D Cell Culture System Minimizes Cellular Stress and Maintains the in Vivo-like Morphological Complexity of Astroglial Cells. Glia 2013, 61 (3), 432-440. [PubMed: 23292921]

(16). Zuidema JM; Hyzinski-García MC; Van Vlasselaer K; Zaccor NW; Plopper GE; Mongin AA; Gilbert RJ Enhanced GLT-1 Mediated Glutamate Uptake and Migration of Primary Astrocytes Directed by Fibronectin-Coated Electrospun Poly-1-Lactic Acid Fibers. Biomaterials 2014, 35 (5), 1439-1449. [PubMed: 24246642]

(17). Johnson CD; D'Amato AR; Puhl DL; Wich DM; Vesperman A; Gilbert RJ Electrospun Fiber Surface Nanotopography Influences Astrocyte-Mediated Neurite Outgrowth. Biomed. Mater 2018, 13 (5), 054101. [PubMed: 29762127]

(18). Wang HB; Mullins ME; Cregg JM; McCarthy CW; Gilbert RJ Varying the Diameter of Aligned Electrospun Fibers Alters Neurite Outgrowth and Schwann Cell Migration. Acta Biomater. 2010, 6 (8), 2970-2978. [PubMed: 20167292] 
(19). Wen X; Tresco PA Effect of Filament Diameter and Extracellular Matrix Molecule Precoating on Neurite Outgrowth and Schwann Cell Behavior on Multifilament Entubulation Bridging Device in Vitro. J. Biomed. Mater. Res., Part A 2006, 76A (3), 626-637.

(20). Simitzi C; Ranella A; Stratakis E Controlling the Morphology and Outgrowth of Nerve and Neuroglial Cells: The Effect of Surface Topography. Acta Biomater. 2017, 51, 21-52. [PubMed: 28069509]

(21). Lee S; Leach MK; Redmond SA; Chong SYC; Mellon SH; Tuck SJ; Feng Z-Q; Corey JM; Chan JR A Culture System to Study Oligodendrocyte Myelination Processes Using Engineered Nanofibers. Nat. Methods 2012, 9 (9), 917-922, DOI: 10.1038/nmeth.2105. [PubMed: 22796663]

(22). Bechler ME; Byrne L; ffrench-Constant C CNS Myelin Sheath Lengths Are an Intrinsic Property of Oligodendrocytes. Curr. Biol 2015, 25 (18), 2411-2416. [PubMed: 26320951]

(23). Debanne D; Campanac E; Bialowas A; Carlier E; Alcaraz G Axon Physiology. Physiol. Rev 2011, 91 (2), 555-602. [PubMed: 21527732]

(24). Zhang H; Hubbard PL; Parker GJM; Alexander DC Axon Diameter Mapping in the Presence of Orientation Dispersion with Diffusion MRI. NeuroImage 2011, 56 (3), 1301-1315. [PubMed: 21316474]

(25). Komlosh ME; Özarslan E; Lizak MJ; Horkayne-Szakaly I; Freidlin RZ; Horkay F; Basser PJ Mapping Average Axon Diameters in Porcine Spinal Cord White Matter and Rat Corpus Callosum Using D-PFG MRI. NeuroImage 2013, 78, 210-216. [PubMed: 23583426]

(26). Remahl S; Hildebrand C Changing Relation between Onset of Myelination and Axon Diameter Range in Developing Feline White Matter. J. Neurol. Sci 1982, 54 (1), 33-45. [PubMed: 7077354]

(27). Yu LMY; Leipzig ND; Shoichet MS Promoting Neuron Adhesion and Growth. Mater. Today 2008, 11 (5), 36-43.

(28). Barnes CP; Sell SA; Boland ED; Simpson DG; Bowlin GL Nanofiber Technology: Designing the next Generation of Tissue Engineering Scaffolds. Adv. Drug Delivery Rev 2007, 59 (14), 14131433.

(29). Elsdale T; Bard J Collagen Substrata for Studies on Cell Behavior. J. Cell Biol 1972, 54 (3), 626637. [PubMed: 4339818]

(30). Qu J; Wang D; Wang H; Dong Y; Zhang F; Zuo B; Zhang H Electrospun Silk Fibroin Nanofibers in Different Diameters Support Neurite Outgrowth and Promote Astrocyte Migration. J. Biomed. Mater. Res., Part A 2013, 101A (9), 2667-2678.

(31). Hurtado A; Cregg JM; Wang HB; Wendell DF; Oudega M; Gilbert RJ; McDonald JW Robust CNS Regeneration after Complete Spinal Cord Transection Using Aligned Poly-1-Lactic Acid Microfibers. Biomaterials 2011, 32 (26), 6068-6079. [PubMed: 21636129]

(32). Perea G; Navarrete M; Araque A Tripartite Synapses: Astrocytes Process and Control Synaptic Information. Trends Neurosci. 2009, 32 (8), 421-431. [PubMed: 19615761]

(33). Roberts RC; Roche JK; McCullumsmith RE Localization of Excitatory Amino Acid Transporters EAAT1 and EAAT2 in Human Postmortem Cortex: A Light and Electron Microscopic Study. Neuroscience 2014, 277, 522-540. [PubMed: 25064059]

(34). Lau C; O’Shea R; Broberg B; Bischof L; Beart P The Rho Kinase Inhibitor Fasudil Up-Regulates Astrocytic Glutamate Transport Subsequent to Actin Remodelling in Murine Cultured Astrocytes. Br. J. Pharmacol 2011, 163 (3), 533-545. [PubMed: 21309758]

(35). O'Shea RD; Lau CL; Zulaziz N; Maclean FL; Nisbet DR; Horne MK; Beart PM Transcriptomic Analysis and 3D Bioengineering of Astrocytes Indicate ROCK Inhibition Produces Cytotrophic Astrogliosis. Front. Neurosci 2015, 9 (50), 1-12. [PubMed: 25653585]

(36). Johnson CDL; D’Amato AR; Gilbert RJ Electrospun Fibers for Drug Delivery after Spinal Cord Injury and the Effects of Drug Incorporation on Fiber Properties. Cells Tissues Organs 2016, 202 (1-2), 116-135. [PubMed: 27701153]

(37). Shenoy SL; Bates WD; Frisch HL; Wnek GE Role of Chain Entanglements on Fiber Formation during Electrospinning of Polymer Solutions: Good Solvent, Non-Specific Polymer-Polymer Interaction Limit. Polymer 2005, 46 (10), 3372-3384.

(38). Western Blot Procedure. Abcam. 
(39). He Z; Jin Y Intrinsic Control of Axon Regeneration. Neuron 2016, 90 (3), 437-451. [PubMed: 27151637]

(40). Koppes AN; Seggio AM; Thompson DM Neurite Outgrowth Is Significantly Increased by the Simultaneous Presentation of Schwann Cells and Moderate Exogenous Electric Fields. J. Neural Eng 2011, 8 (4), 1-13.

(41). Croce N; Bernardini S; Di Cecca S; Caltagirone C; Angelucci F Hydrochloric Acid Alters the Effect of L-Glutamic Acid on Cell Viability in Human Neuroblastoma Cell Cultures. J. Neurosci. Methods 2013, 217 (1-2), 26-30. [PubMed: 23612442]

(42). Biran R; Noble MD; Tresco PA Directed Nerve Outgrowth Is Enhanced by Engineered Glial Substrates. Exp. Neurol 2003, 184 (1), 141-152. [PubMed: 14637087]

(43). Beller JA; Gurkoff GG; Berman RF; Lyeth BG Pharmacological Enhancement of Glutamate Transport Reduces Excitotoxicity in Vitro. Restor. Neurol. Neurosci 2011, 29 (5), 331-346. [PubMed: 21846950]

(44). Wang Z-J; Liang C-L; Li G-M; Yu C-Y; Yin M Neuroprotective Effects of Arachidonic Acid against Oxidative Stress on Rat Hippocampal Slices. Chem.-Biol. Interact 2006, 163 (3), 207217. [PubMed: 16982041]

(45). Ziemińska E; Stafiej A; Łazarewicz JW Role of Group I Metabotropic Glutamate Receptors and NMDA Receptors in Homocysteine-Evoked Acute Neurodegeneration of Cultured Cerebellar Granule Neurones. Neurochem. Int 2003, 43 (4), 481-492. [PubMed: 12742095]

(46). Chen C-J; Liao S-L; Kuo J-S Gliotoxic Action of Glutamate on Cultured Astrocytes. J. Neurochem 2000, 75 (4), 1557-1565. [PubMed: 10987836]

(47). Nissim I Newer Aspects of Glutamine/Glutamate Metabolism: The Role of Acute PH Changes. Am. J. Physiol. - Ren. Physiol 1999, 277 (4), F493-F497.

(48). Al Awabdh S; Gupta-Agarwal S; Sheehan DF; Muir J; Norkett R; Twelvetrees AE; Griffin LD; Kittler JT Neuronal Activity Mediated Regulation of Glutamate Transporter GLT-1 Surface Diffusion in Rat Astrocytes in Dissociated and Slice Cultures. Glia 2016, 64 (7), 1252-1264. [PubMed: 27189737]

(49). East E; de Oliveira DB; Golding JP; Phillips JB Alignment of Astrocytes Increases Neuronal Growth in Three-Dimensional Collagen Gels and Is Maintained Following Plastic Compression to Form a Spinal Cord Repair Conduit. Tissue Eng., Part A 2010, 16 (10), 3173-3184. [PubMed: 20649441]

(50). Nguyen AT; Sathe SR; Yim EKF From Nano to Micro: Topographical Scale and Its Impact on Cell Adhesion, Morphology and Contact Guidance. J. Phys.: Condens. Matter 2016, 28 (18), 116.

(51). Kilinc D; Blasiak A; Lee GU Microtechnologies for Studying the Role of Mechanics in Axon Growth and Guidance. Front. Cell. Neurosci 2015, 9, 1-8. [PubMed: 25667569]

(52). Norris CR; Kalil K Guidance of Callosal Axons by Radial Glia in the Developing Cerebral Cortex. J. Neurosci 1991, 11 (11), 3481-3492. [PubMed: 1941093]

(53). Kalil K; Dent EW Branch Management: Mechanisms of Axon Branching in the Developing Vertebrate CNS. Nat. Rev. Neurosci 2014, 15 (1), 7-18. [PubMed: 24356070]

(54). Lepore AC; O’Donnell J; Bonner JF; Paul C; Miller ME; Rauck B; Kushner RA; Rothstein JD; Fischer I; Maragakis NJ Spatial and Temporal Changes in Promoter Activity of the Astrocyte Glutamate Transporter GLT1 Following Traumatic Spinal Cord Injury. J. Neurosci. Res 2011, 89 (7), 1001-1017. [PubMed: 21488085]

(55). Lepore AC; O’Donnell J; Kim AS; Yang EJ; Tuteja A; Haidet-Phillips A; O’Banion CP; Maragakis NJ Reduction in Expression of the Astrocyte Glutamate Transporter, GLT1, Worsens Functional and Histological Outcomes Following Traumatic Spinal Cord Injury. Glia 2011, 59 (12), 1996-2005. [PubMed: 21882244]

(56). Falnikar A; Hala TJ; Poulsen DJ; Lepore AC GLT1 Overexpression Reverses Established Neuropathic Pain-Related Behavior and Attenuates Chronic Dorsal Horn Neuron Activation Following Cervical Spinal Cord Injury. Glia 2016, 64 (3), 396-406. [PubMed: 26496514]

(57). Li K; Nicaise C; Sannie D; Hala TJ; Javed E; Parker JL; Putatunda R; Regan KA; Suain V; Brion J-P; Rhoderick R; Wright MC; Poulsen DJ; Lepore AC Overexpression of the Astrocyte Glutamate Transporter GLT1 Exacerbates Phrenic Motor Neuron Degeneration, Diaphragm 
Compromise, and Forelimb Motor Dysfunction Following Cervical Contusion Spinal Cord Injury. J. Neurosci 2014, 34 (22), 7622-7638. [PubMed: 24872566]

(58). Ugbode CI; Hirst WD; Rattray M Astrocytes Grown in Alvetex ${ }^{\circledR}$ Three Dimensional Scaffolds Retain a Non-Reactive Phenotype. Neurochem. Res 2016, 41 (8), 1857-1867. [PubMed: 27099962]

(59). Danbolt NC Glutamate Uptake. Prog. Neurobiol 2001, 65 (1), 1-105. [PubMed: 11369436] 
Film

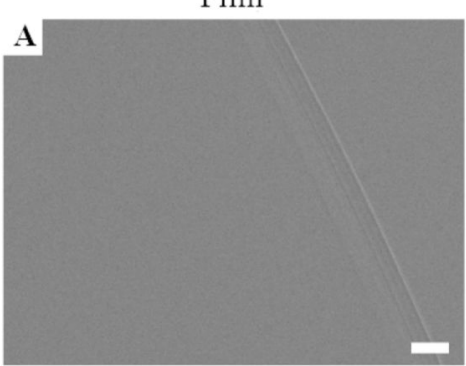

D

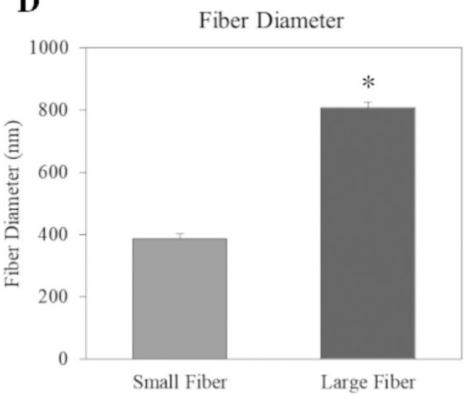

Small Fiber

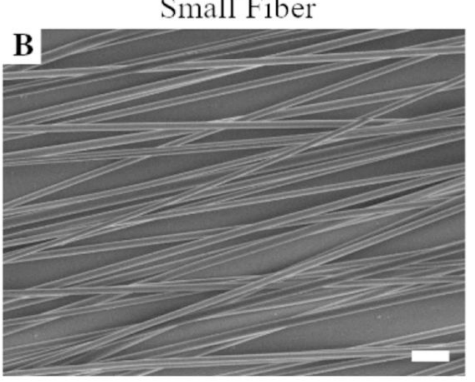

E

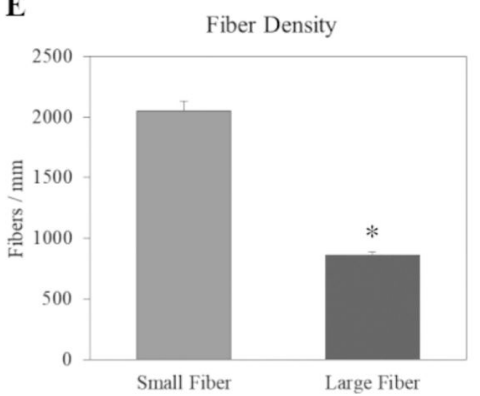

Large Fiber
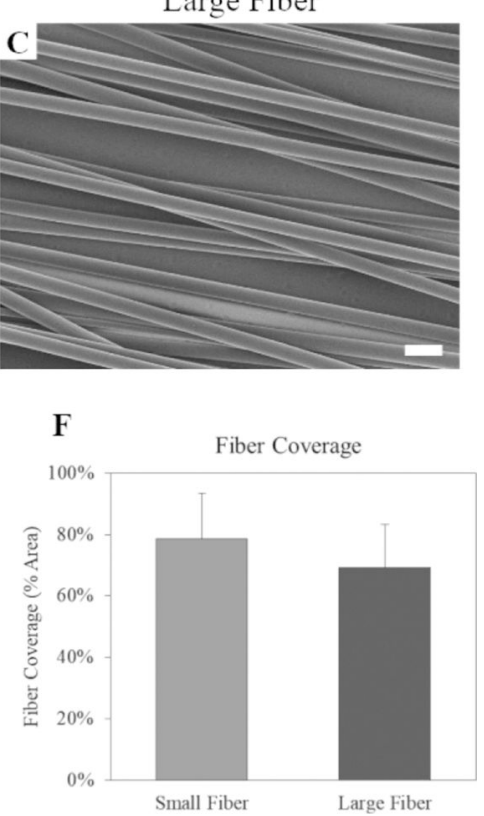

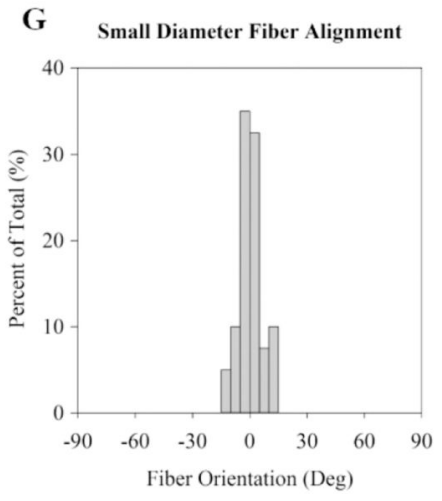

H Large Diameter Fiber Alignment

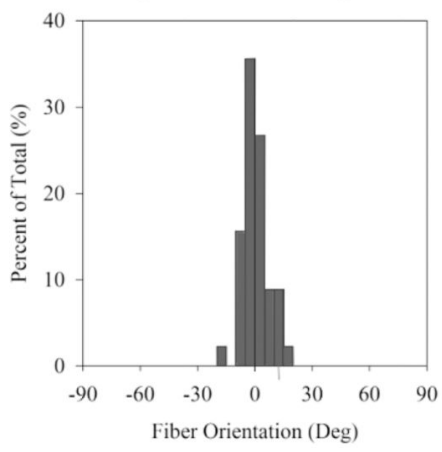

Figure 1.

Characterization of small (7\% PLLA) and large (12\% PLLA) electrospun fibers. Scanning electron micrographs of (A) film, (B) small diameter fibers, and (C) large diameter fiber surfaces. Scale bar $=2 \mu \mathrm{m}$. (D) The scaffold's mean fiber diameter. (E) Fiber density or the fibers per mm on each scaffold. (F) The fiber surface coverage. The alignment of the $(\mathrm{G})$ small fibers and $(\mathrm{H})$ large fibers. The * indicates statistical significance $(p<0.05)$. Statistical differences in fiber alignment were not observed. 
A

A $_{10}$

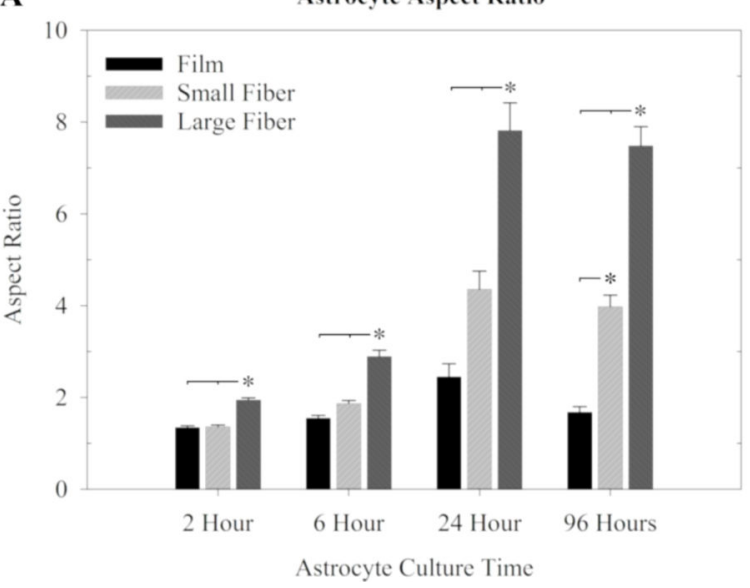

1 Day
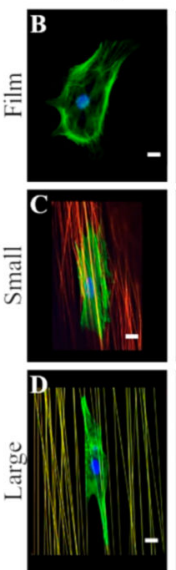

4 Day

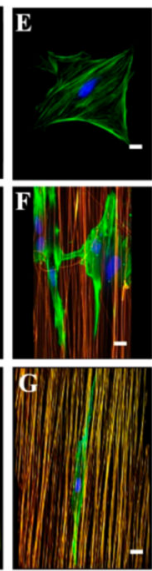

Figure 2.

Astrocyte elongation over time on film, small diameter fiber, and large diameter fiber substrates. (A) Astrocyte aspect ratio (length/ width) on large fiber, small fiber, or film substrates after $2 \mathrm{~h}, 6 \mathrm{~h}, 24 \mathrm{~h}$ ( 1 day), and $96 \mathrm{~h}$ (4 days) in culture on the surfaces. The * indicates statistical significance (ANOVA, $p \leq 0.01$ ). (B-G) Astrocytes from selected time points are shown on fluorescent fibers: (B-D) 1 day and (E-G) 4 day (green, phalloidin; blue, DAPI; red, rhodamine labeled fibers; scale $=20 \mu \mathrm{m}$ ). 

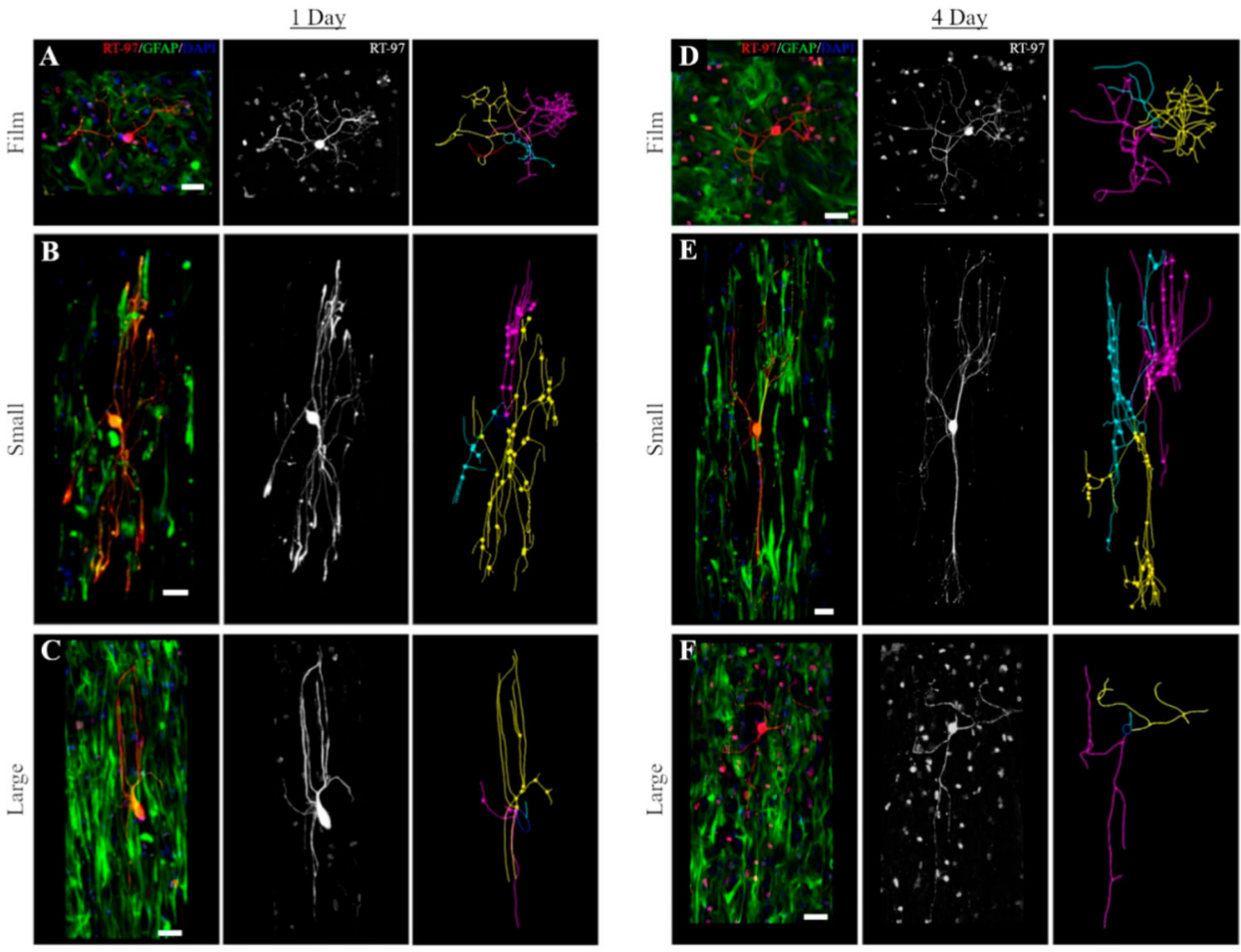

G

Neurite Outgrowh on 1 Day Astrocytes

Neurite Outgrowh on 4 Day Astrocytes
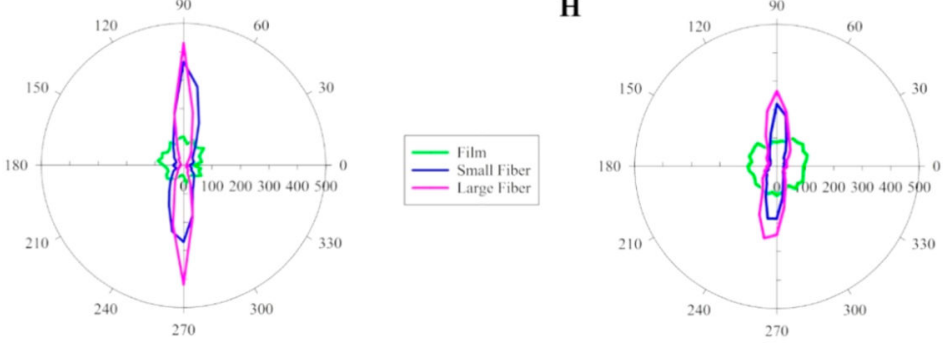

I
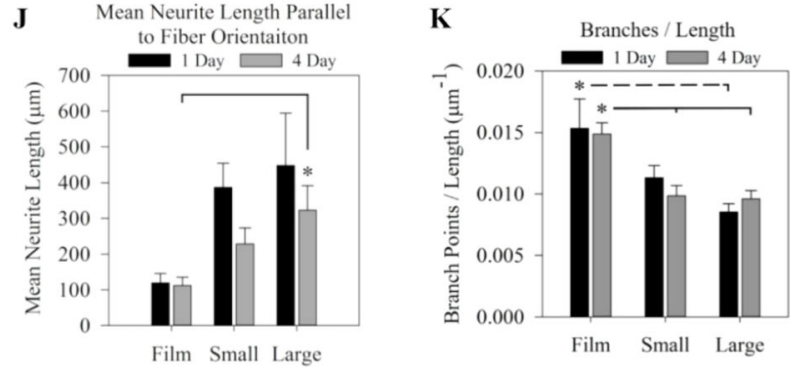

Figure 3.

Effects of fiber diameter on astrocyte-mediated neurite guidance. Astrocytes cultured on electrospun fiber surfaces for 1 day (A-C) or 4 days (D-F) on either film (A,D), small diameter fibers $(\mathrm{B}, \mathrm{E})$, or large diameter fibers $(\mathrm{C}, \mathrm{F})$ before neurons were introduced for $24 \mathrm{~h}$ (for timeline, see I). Panels are arranged to show the coculture in the first column (green, GFAP; red, RT-97; blue, DAPI), the neurons alone in the second column, and the tracing of the neurites in the third column. Scale $=50 \mu \mathrm{m}$. Polar plots show the mean neurite outgrowth length in each direction for astrocytes cultured for either $(\mathrm{G}) 1$ day or $(\mathrm{H}) 4$ days on each 
substrate. The fiber orientation was parallel to the 90 deg axis. (J) The mean neurite growth length parallel to the orientation of the fibers on each astrocyte surface over time. (K) The number of neurite branches per micrometer in the neuron-astrocyte coculture on each surface over time. The * represents statistical significance (ANOVA, $p \leq 0.05$ ). 
A
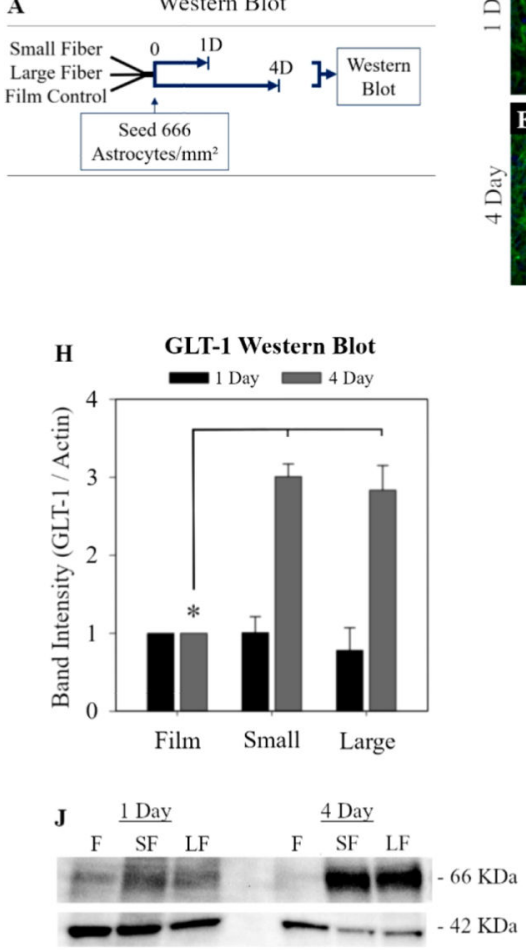

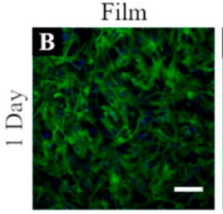

Small
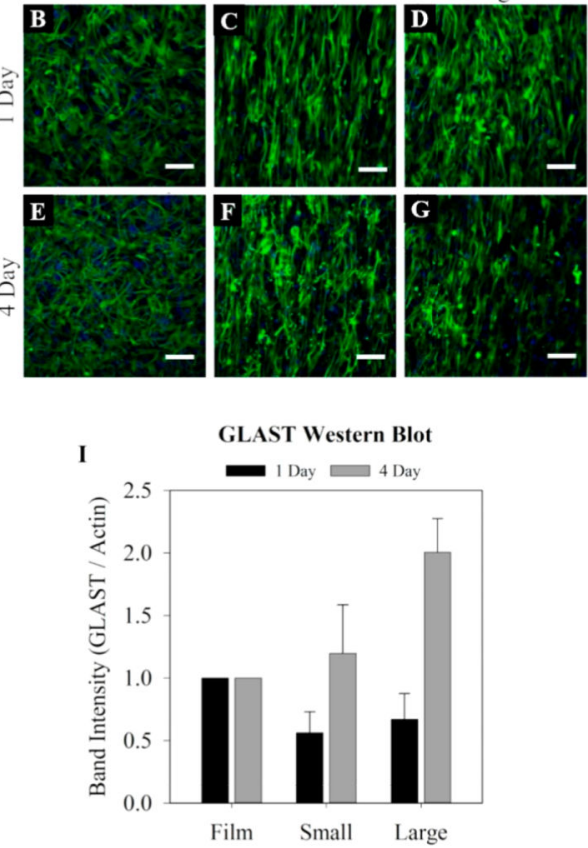

$\mathbf{K}$

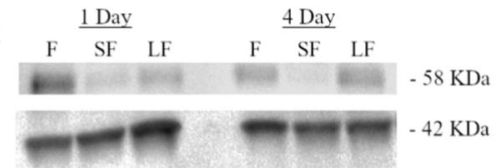

Figure 4.

Effect of fiber diameter on astrocyte glutamate transporters. The relative amounts of GLT-1 $(66 \mathrm{kDa})$ and GLAST $(52 \mathrm{kDa})$ was assessed using Western blot. GLT-1 and GLAST were normalized to actin (42 kDa). (A) Diagram of the Western blot protocol (B-G). Higher density astrocytes were seeded for Western blot $\left(666\right.$ cells $/ \mathrm{mm}^{2}$, scale $\left.=100 \mu \mathrm{m}\right)$.

Normalized Western blot results are shown as a fold change over the film control for GLT-1 $(\mathrm{H}, \mathrm{J})$ and GLAST (I, K). The * represents statistical significance (ANOVA, $p \leq 0.05$ ). 
A Glutamate Excitotoxicity

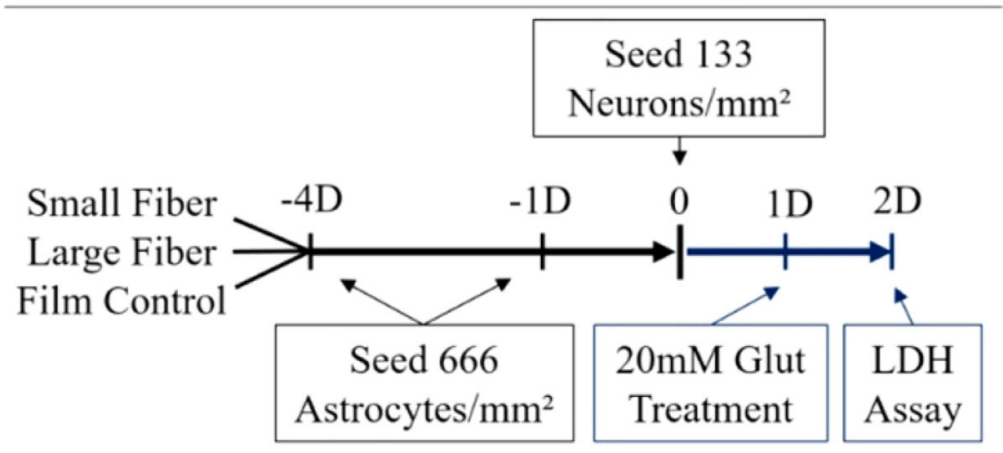

B

\% Survival After Excitotoxicity

1 Day $\square$ Day

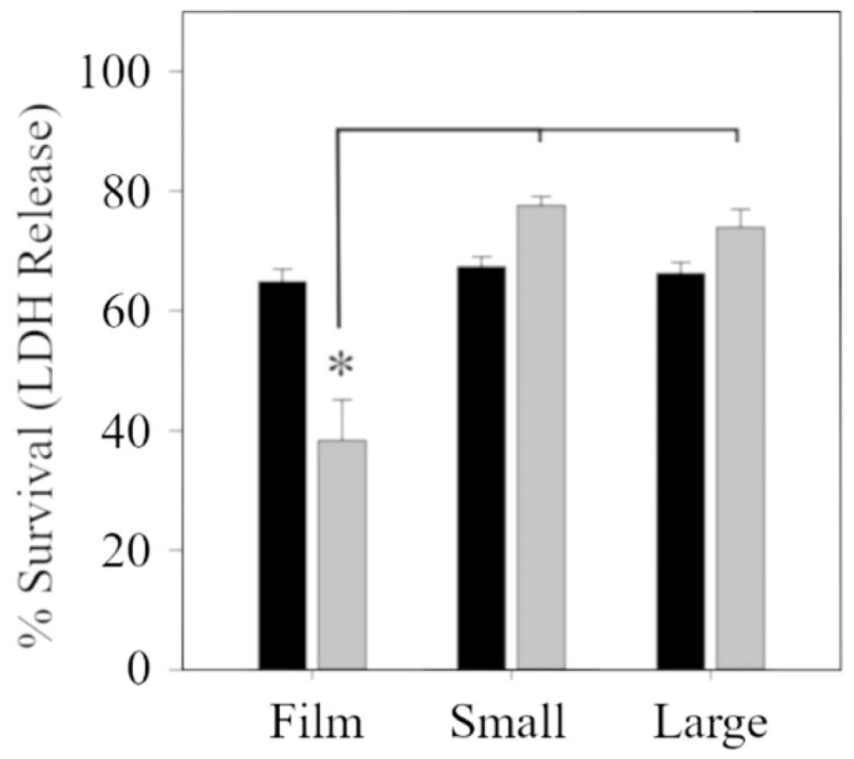

Figure 5.

Fiber induced astrocyte-mediated neuroprotection. (A) Diagram of the excitotoxicity protocol. Cocultures were prepared similar to previous experiments, then exposed to $20 \mathrm{mM}$ glutamate for a period of $24 \mathrm{~h}$. LDH release was used to measure cell death. (B) The \% survival of cocultures after either 1 day or 4 days of astrocyte culture on each of the surfaces. The * represents statistical significance (ANOVA, $p \leq 0.05$ ). 


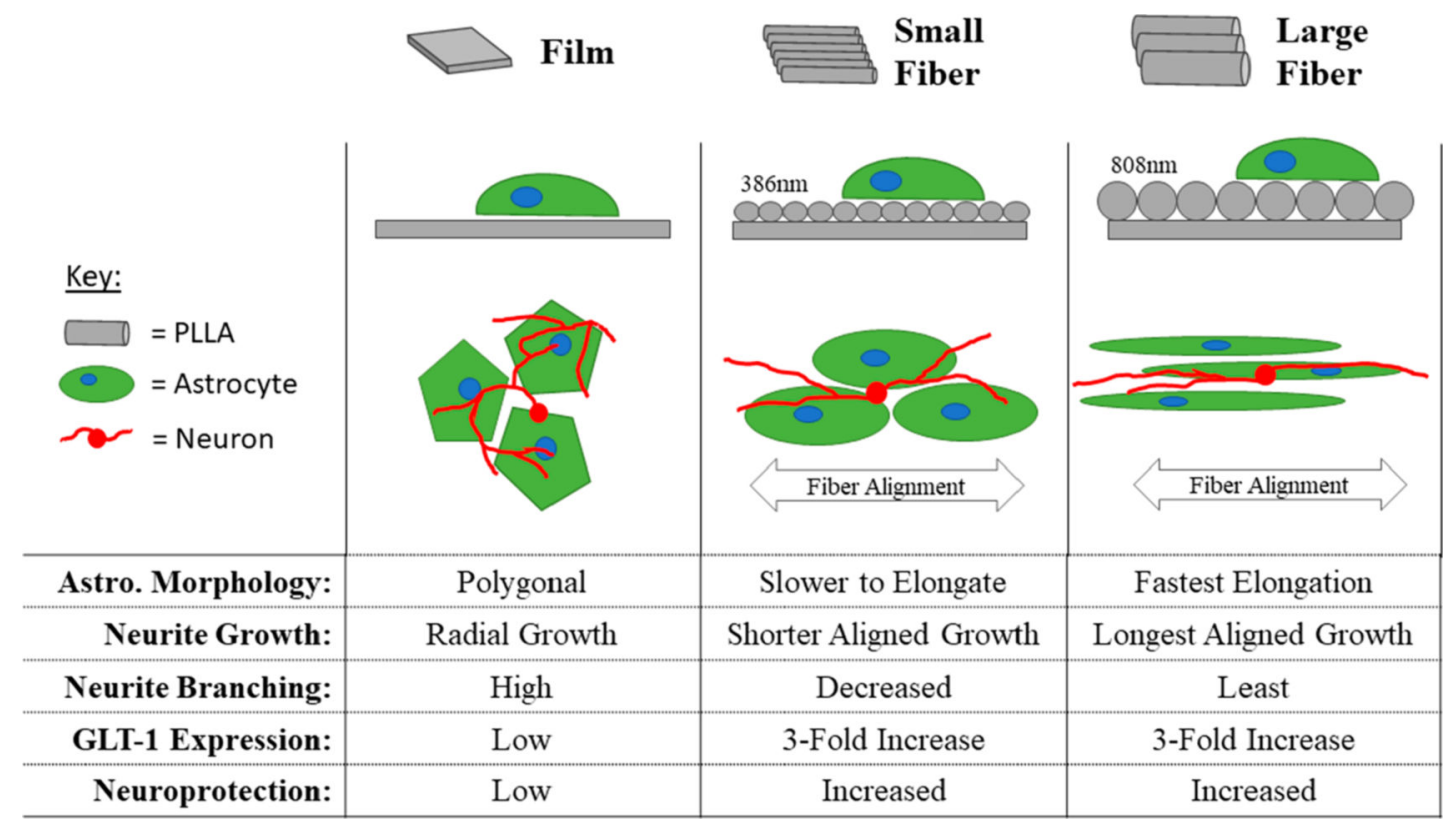

Figure 6.

Summary of astrocyte and astrocyte/neuron response to fiber diameter. In the orthogonal view in the second row, the fibers are drawn in gray. The large fibers are twice the diameter of the small fibers, and the model astrocytes (green) are the same size. In the third row, representative astrocytes are drawn in green, and neurons are drawn in red (not to scale). 

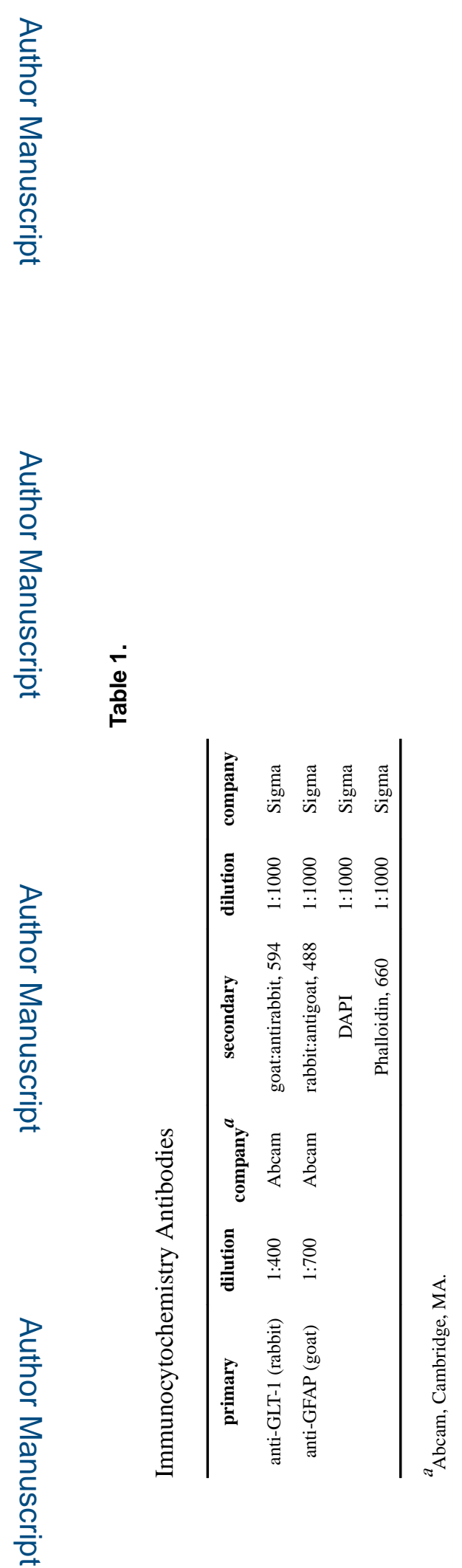

ACS Appl Bio Mater. Author manuscript; available in PMC 2020 January 22. 


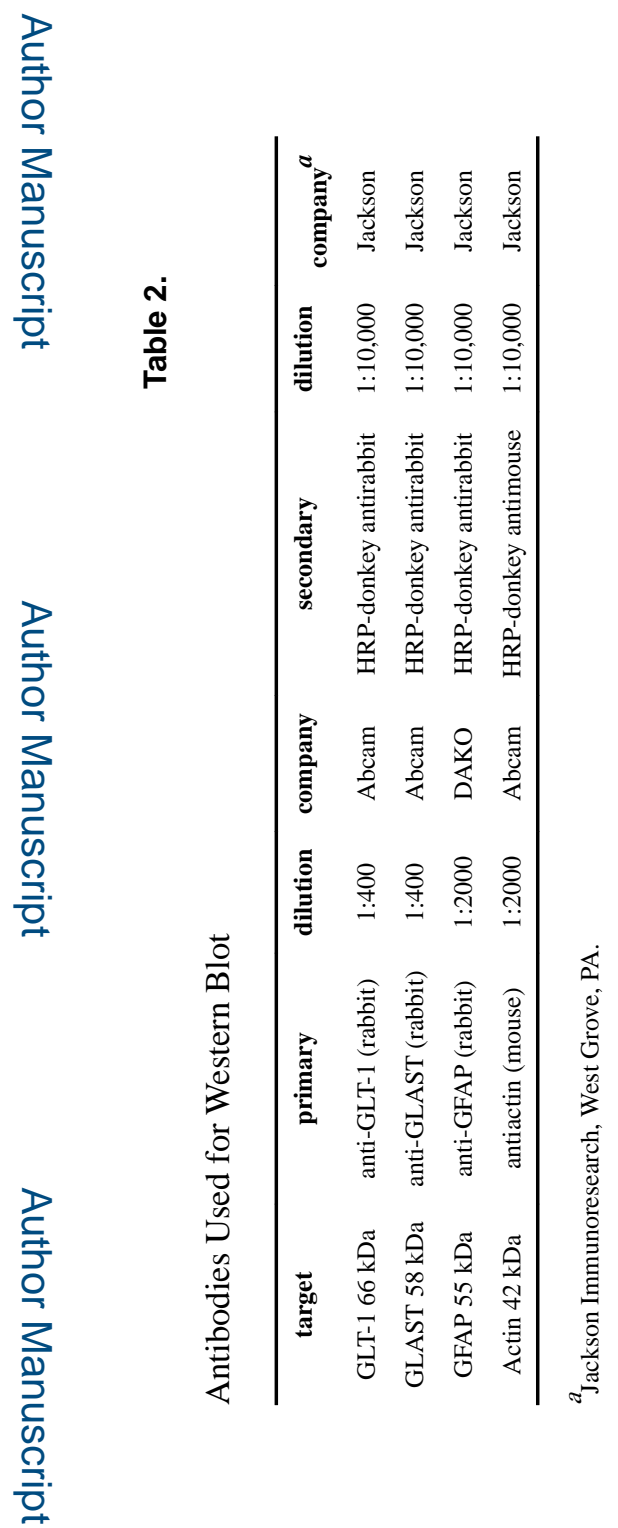

ACS Appl Bio Mater. Author manuscript; available in PMC 2020 January 22. 
Table 3.

Adjusted Electrospinning Parameters

\begin{tabular}{lcc}
\hline \multicolumn{1}{c}{ parameter } & large fiber & small fiber \\
electrospinning solution content & $12 \%$ PLLA & $7 \%$ PLLA \\
rotation speed & $1000 \mathrm{rpm}$ & $2000 \mathrm{rpm}$ \\
pump rate & $2.0 \mathrm{~mL} / \mathrm{h}$ & $1.5 \mathrm{~mL} / \mathrm{h}$ \\
humidity & $38-40 \%$ & $30-33 \%$ \\
collection time & $10 \mathrm{~min}$ & $15 \mathrm{~min}$ \\
\hline
\end{tabular}

